\title{
Age-related Dysregulation of Autophagy Contributes to Microglial Dysfunction and Chronic Neurobehavioral Deficits After Traumatic Brain Injury
}

\section{Rodney Ritzel}

University of Maryland School of Medicine https://orcid.org/0000-0002-0160-2930

Yun Li

University of Maryland School of Medicine

Zhuofan Lei

University of Maryland School of Medicine Jordan Carter

University of Maryland School of Medicine Junyun $\mathrm{He}$

University of Maryland School of Medicine Harry Choi

University of Maryland School of Medicine

Niaz Khan

University of Maryland School of Medicine

Hui Li

University of Maryland School of Medicine

Samantha Allen

University of Maryland School of Medicine

Marta M Lipinski

University of Maryland School of Medicine

Alan I Faden

University of Maryland School of Medicine

Junfang Wu ( $\square$ junfang.wu@som.umaryland.edu )

University of Maryland School of Medicine https://orcid.org/0000-0003-3338-7291

\section{Research article}

Keywords: Aging, Autophagy, Microglia, Neurodegeneration, Neuroinflammation, Traumatic brain injury

Posted Date: November 11th, 2021 
DOI: https://doi.org/10.21203/rs.3.rs-1021695/v1

License: (c) (1) This work is licensed under a Creative Commons Attribution 4.0 International License. Read Full License 


\section{Abstract}

Background: Elderly patients with traumatic brain injury (TBI) have greater mortality and poorer outcomes than younger individuals. Given the critical role for autophagy in promoting the cellular degradation of damaged organelles and the negative impact of aging on this protective mechanism, we hypothesized that treatment with an autophagic inducer such as trehalose may be a promising therapeutic strategy for TBI aged animals.

Methods: Controlled cortical impact was used to induce moderate TBI in male C57BL/6 mice. Young (10 weeks) or old (18 months) animals were evaluated behaviorally for 12 weeks after injury. Autophagy, inflammation, and neurodegeneration were examined using NanoString analysis, flow cytometry, and immunohistochemistry. Trehalose or sucrose as a control were administered in drinking water beginning day1 post-injury for 8 weeks.

Results: Aged mice had a higher mortality rate than young mice at 12 weeks post-injury. At 12 weeks, aged mice showed greater cognitive deficits and depression than younger animals. There were also larger lesion volumes and increased microglial activation in aged TBI mice at 16 weeks post-injury. NanoString analysis identified several age- and TBI-specific genes changes that were differentially expressed in the chronically injured brain, including those involved with the complement, phagocytosis, and autophagy pathways. Flow cytometry demonstrated temporal dysregulation of phagocytosis and autophagy in microglia from injured mice that were exacerbated with age and accompanied by an increased inflammation and lymphocyte infiltration. Old TBI mice treated with trehalose exhibited either more delayed deficits or enhanced recovery in cognitive and motor tasks. In addition, Trehalose treatment modified expression of autophagy markers and attenuated the microglial response to TBI.

Conclusions: Our data indicate that microglia undergo chronic changes in autophagic regulation with both normal aging and TBI that are associated with poorer functional outcome. Enhancing autophagy may therefore be a promising clinical therapeutic strategy for TBI, especially in older patients.

\section{Background}

Neurodegenerative disease in the elderly is a major healthcare challenge and growing economic burden. Traumatic brain injury (TBI), a leading cause of death among young people, is increasingly prevalent in the aging population. Millions of TBI survivors live with varying degrees of disability and have an increased risk for developing neurodegenerative disease such as Alzheimer's disease (AD), Parkinson's disease and chronic traumatic encephalopathy $[1,2]$. Elderly TBI patients have higher mortality and greater disability than younger individuals, and are more likely to develop a neurodegenerative disorder [35]. For these reasons, older subjects are often excluded from clinical trials and treated less aggressively [6].

Our knowledge of TBI pathophysiology in aged animals is limited, and our understanding of the secondary injury mechanisms that affect long-term outcome remains limited. Whereas direct targeting of 
inflammatory pathways in old mice appears beneficial in experimental settings [7, 8], therapeutic intervention of certain other proposed injury mechanisms, such as autophagy [9], have not been as well addressed in older animals. Both normal aging and the chronic phase of TBI are associated with a basal elevation in microglial activation and neuroinflammation. Chronic activation of microglia has been linked to alterations in cellular functions essential for survival [10]. Among these, autophagy is a process that degrades and recycles cytoplasmic proteins and organelles; and it plays a critical role in inflammation, the homeostasis of microglia, and plaque development in $\operatorname{AD}[11,12]$. Autophagic mechanisms have been found to be impaired with age and during acute brain injury $[13,14]$. However, whether aging and TBI synergistically interact to disrupt autophagic function in microglia, aggravate neuroinflammation, and hamper long-term neurological recovery is still unclear.

Despite recent funding and research efforts towards understanding age-related neurodegenerative disease, the secondary injury mechanisms that account for the age-related disparity in TBI outcomes remain, including cognitive dysfunction, have been insufficiently examined. Given the limited pre-clinical studies examining injury mechanisms and related outcomes in experimental injury models using aged animals $[15,16]$, although endogenous recovery mechanisms are impaired with aging, these pathways may still remain amenable to therapeutic modulation. Here we examine the hypothesis that age-related defects in autophagic function can exacerbate chronic microglial activation, neurodegeneration, and long-term functional recovery in old mice which can be partially rescued by improving autophagic function.

\section{Methods}

\section{Animals and TBI model}

All surgical procedures and animal experiments were performed according to the protocols approved by the University of Maryland School of Medicine Institutional Animal Care and Use Committee (IACUC). Experiments were conducted using young adult male C57BL/ 6 mice (10-12 weeks old), or aged male mice (18 months old) from Charles River. After being fully anesthetized with isoflurane, mice were subjected to either controlled cortical injury (CCl) or sham surgery $[17,18]$. Briefly, a midline incision of approximately $10-\mathrm{mm}$ in length was made over the skull, with the skin and fascia retracted, and a 4-mm craniotomy was made on the central aspect of the left parietal bone. An injury of moderate severity was induced by a TBI-0310 Head Impactor (Precision Systems and Instrumentation) with a 3.5-mm diameter tip followed by impact velocity of $3.9 \mathrm{~m} / \mathrm{s}$ and a displacement depth of $1.2 \mathrm{~mm}$. After surgery, all mice were assigned to one of four groups based on surgery (sham or TBI) and treatment (trehalose or sucrose control) according to a randomized block experimental design. The surgical procedures were performed by the same investigator and all behavioral tests were carried out with the same equipment by blinded experimenters.

\section{Experimental design}


Study 1: To investigate whether age affects neurological function and pathological changes after TBI, young or aged C57BL/6 male mice were subjected to either moderate CCl or sham surgery (Fig 1a). All mice underwent a battery of neurobehavioral tasks which consisted of open field (OF), Catwalk (CW), grip strength (GS), and rotarod for assessment of motor function; Y-maze (YM) and novel object recognition (NOR) for cognitive function; novelty suppressed feeding (NSF) and social recognition (SR) for depressive-like behaviors; and hot plate for pain sensitivity. The OF, YM, CW, GS, rotarod and HP tests were performed starting at 4 days before $\mathrm{CCl}$ for establishment of functional performance baseline between young and old mice and repeatedly at 1 week, 3, 6, and 9 weeks after $\mathrm{CCl}$. At 12 weeks postinjury, a final round of behavior experiments was conducted to assess motor and cognitive function, in addition to depressive-like behaviors. After completion of all behavioral tests, ipsilateral brain tissue was collected at 16 weeks post-injury and processed for NanoString analysis, flow cytometry assays, and histological outcome measures.

Study 2: To assess whether autophagy enhancer mitigates age-exacerbated functional recovery after $\mathrm{TBI}$, aged C57BL/ 6 mice were fed trehalose or sucrose as vehicle starting on the date of the injury until the time of sacrifice. Based on prior studies [19-21] and our pilot data, a single dose of $5 \%$ trehalose (Sigma-Aldrich, Cat\# T9449) or 5\% sucrose as vehicle control was injected subcutaneously into each mouse immediately after $\mathrm{CCl}$ surgery. $5 \%$ trehalose or sucrose was also administered into their drinking water for the first 7 days followed by continuous administration of trehalose or sucrose solution at $2.5 \%$ of concentration in their drinking water. The OF, YM, CW, GS, and rotarod tests were performed starting at 4 days before $\mathrm{CCl}$ and repeatedly at 2 and 4 weeks after $\mathrm{CCl}$. At 6 weeks post-injury, a final round of behavior experiments was conducted to fully assess the effects of trehalose on functional recovery after TBI. After completion of all behavioral tests, ipsilateral brain tissue was collected at 9 weeks post-injury and processed for flow cytometry assays.

\section{Neurological behavioral tests}

The following behavior tests were performed with mice and group information blinded to the operators. To minimize stress and fatigue, each test was performed on a different day.

\section{Motor function}

Open field (OF) test: Spontaneous locomotor activity was examined in the OF apparatus [22]. Each test subject mouse was individually placed in a corner while facing towards the chamber wall of the apparatus $(22.5 \times 22.5 \mathrm{~cm})$. The mice were allowed to freely explore the chambers for $10 \mathrm{~min}$. Parameters on the distance travelled, average speed, time immobile and percentage of time spent in the center of the chamber were recorded by the computer-based ANY-maze automated video-tracking system (Stoelting Co).

Gait analysis with CatWalk XT (CW): Analysis of gait and posture was performed with the CatWalk XT automated system as mentioned in our previous publications (Noldus; RRID:SCR_004074) [17, 18, 23]. Acquisition of data took place in a darkened room with red light. A single researcher blinded to the 
grouping of each mouse was tasked with handling the subjects. The Catwalk apparatus records print position, gait postures, and weight distribution through its green illuminated walkway. A minimum of 3 valid runs, complete crossings with no turns or scaling of sidewalls, were obtained for each tested mouse. Runs that didn't comply to the preset standards were excluded from the final analysis.

Rotarod test: The accelerating Rotarod was used to assess locomotor function and coordination at baseline and various timepoints post-injury [24]. The mouse was placed on a rotarod device (IITC Life Science, Inc.), and their latency to falling off the accelerating rotarod was recorded. The acceleration settings for the device were 4 to $40 \mathrm{rpm}$ over 90 seconds (s), with each trial lasting for a maximum of 300 s. Individual scores from three trials were averaged and evaluated relative to their baseline latencies.

Grip strength (GS) test: Grip strength was measured using a digital grip strength meter (Bioseb BP, In Vivo Research Instruments, France), as previously described [25]. Forelimb grip strength was measured from the mouse using both the ipsilateral and contralateral forepaws together. The mouse was held by its tail, the forelimbs were placed on the grasping metal wire grid and the mouse gripped the wire grid attached to the force transducer. Once the grip was secured, the animal was slowly pulled away from the bar. The maximal average force exerted on the grip strength meter by both forepaws was averaged from 10 trials per day for each mouse at each timepoint.

\section{Cognitive function}

Y-maze (YM) test: YM was used to assess the hippocampus-dependent spatial working memory of mice as described previously $[17,18]$. The percentage of spontaneous alterations was calculated using the following equation: total alternations $\times 100$ /(total arm entries - 2). If a mouse had a percentage of alternation above $50 \%$ (the chance level for randomly choosing the unfamiliar arm), it was indicative of a functional spatial memory.

Novel object recognition (NOR): For testing non-hippocampal mediated memory, mice underwent NOR according to procedures described in previous studies $[17,18]$. Mice were tested in an open field apparatus after a 5 min habituation period on the first day. The time spent with two identical objects was recorded on the second day of testing, and one of the familiar objects were switched out with a novel object on the third day. Testing stopped after each mouse went through a sum total of $30 \mathrm{~s}$ exploration time. Since mice would inherently prefer to explore novel objects, a preference for the novel object with an exploration time of more than $15 \mathrm{~s}$ was considered as having intact learning and memory skills.

\section{Depressive-like behaviors}

Novelty-suppressed feeding (NSF) test: The NSF was performed on subject mice to assess a rodent's aversion to eating in a novel environment. After fasting for $24 \mathrm{~h}$, mice were placed in an open field apparatus designed to model the introduction of a novel environment. Several food pellets were placed in the center of the apparatus and latency time for each mouse to reach the food and bite into it was 
recorded. If the mouse failed to eat within the maximum time of $10 \mathrm{~min}$, a latency time of $600 \mathrm{~s}$ was recorded for this subject.

Social recognition (SR) test: This task is based on rodent's innate tendency to investigate a novel congener over a familiar one $[17,18,26]$. Using a three-chambered rectangular apparatus made of Plexiglas with each one at equal size $(20 \times 40 \times 23 \mathrm{~cm})$. An opening between the walls allows for free access to each chamber, which contains two identical wire mesh cup containers. Before testing, each mouse was single-housed overnight. On the first day, the tested mice were placed in the apparatus with two empty cups for a 10 min habituation period. On the second day, a stranger mouse was introduced and randomly placed inside one of the empty cups in either the left- or right-side chamber while the other cup was left empty. The tested mouse started from the middle chamber and allowed to freely explore all three chambers for an exploration period of $10 \mathrm{~min}$. Afterwards, a second unfamiliar, stranger was placed inside the previously empty cup. The test subject was once again allowed to freely explore all three chambers for a period of $10 \mathrm{~min}$. Exploration time that the subject mice spent with each cup versus stranger mouse was recorded. Since a socially functional mouse would naturally seek out unfamiliar mice for interaction, the test subject was considered capable of social recognition if index for novel mouse scored higher than $50 \%$.

\section{Thermal stimulation}

Hot plate (HP) test: To test pain sensitivity of the hindpaws, mice were placed on the contact probe of computerized thermal stimulator on an Incremental Hot/Cold Plate Analgesia Meter (PE34, IITC Life Science, Woodland Hills, CA). The temperature was increased from $30 \otimes \mathrm{C}$ to $50 \llbracket \mathrm{C}$ with the incremental rate at $10 \otimes C$ per minute. When the tested mouse licked either one of its hindpaws, the test was stopped, and the threshold temperature was recorded. The test was conducted twice with the interval of $3 \mathrm{~h}$ [27].

\section{NanoString neuroinflammation panel analysis}

Following completion of all behavioral tests, mice were euthanized, and RNA samples were obtained from the ipsilateral cerebral cortex surrounding the injury site and the ipsilateral hippocampus. Total RNA (20 $\mathrm{ng} / \mathrm{ul}$ ) was run on a NanoString nCounter system using the Mouse Neuroinflammation v1.0 panel (NanoString Technologies, Seattle, WA) to profile RNA transcript counts for 757 genes and 13 housekeeping genes $[28,29]$. The transcription counts were normalized prior to downstream analysis and pairwise differential expression analysis with the NanoString nSolver software Version 4.0. All statistical analysis of NanoString data was performed in R language software RStudio Version 1.2.5033. Principle component analysis (PCA) was performed with the command 'prcomp' and a Euclidean distance measurement method was used for clustering. The four pairwise mentioned in this article are as follows: 1) Old Sham vs. Young Sham; 2) Young TBI vs. Young Sham; 3) Old TBI vs. Old Sham; 4) Old TBI vs. Young TBI. All pairwise comparisons of 'A vs. B' should be interpreted as 'A relative to B' in the text and figures.

\section{RNA extraction and qPCR}


Total RNA was extracted from flash frozen tissue samples with the miRNeasy Mini Kit (Qiagen, Cat\# 74104). Complementary DNA (cDNA) was synthesized with the Verso cDNA RT kit (Thermo Scientific, Cat\# AB1453B). Both kits were used according to the manufacturer's instructions included in the kit box. Quantitative PCR for all target RNAs (see Table 1) was performed with the TaqMan gene expression assay kit (Applied Biosystems). Each sample was run in duplicates with 3 stages of 40 cycles, 2 min at $50^{\circ} \mathrm{C}, 10 \mathrm{~s}$ at $95^{\circ} \mathrm{C}$ (denaturing step) followed by a final transcription step of $1 \mathrm{~min}$ at $60^{\circ} \mathrm{C}$. Gene expression was normalized by the transcription counts of GAPDH and final relative expression levels were calculated with the $2-\Delta \Delta \mathrm{Ct}$ method $[28,29]$.

\section{Lesion volume, immunohistochemistry (IHC), and quantification}

A subset of tested mice was perfused intracardially with normal saline followed by $4 \%$ paraformaldehyde. The brain was extracted and embedded in Tissue-Tek OCT compound (Sakura, Cat\# 4583). Serial sections of $20 \mathrm{~mm}$ and $60 \mathrm{~mm}$ thickness were placed on Superfrost Plus slides (ThermoFisher, Cat\# 4951PLUS). Lesion volume was measured on $60 \mathrm{~mm}$ coronal sections that were stained with cresyl violet (FD NeuroTechnologies, Cat\# PS102-02). Quantification of the lesion volume was performed with the Stereoinvestigator software (MBF Biosciences). By outlining the missing tissue on the injured hemisphere, the software was able to estimate lesion volume with the Cavalieri method at a grid spacing of $0.1 \mathrm{~mm}[18]$.

Immunofluorescence imaging was performed on $20 \mathrm{~mm}$ coronal brain sections at around $-1.70 \mathrm{~mm}$ from bregma using standard immunostaining protocol, as described previously $[22,28]$. Briefly, sections were blocked with $5 \%$ goat or guineapig serum containing $0.3 \%$ Triton $X-100$. After incubation with primary and secondary antibodies (see Table 1), sections were counterstained with 4',6-diamidino-2-phenylinodole (DAPI, Sigma-Aldrich, Cat\# MBD0015) and mounted with glass coverslips using an anti-fade Hydromount solution (National Diagnostics, Cat\# HS106100ML). Images ( $\mathrm{n}=6$ sections/location/mouse for 5-6 mice/group) were acquired using a fluorescent Nikon Ti-E inverted microscope, at 20X (CFI Plan APO VC 20X NA 0.75 WD $1 \mathrm{~mm}$ ) magnification and the background of each image was subtracted using background $\mathrm{ROI}[30,31]$. The number of $\mathrm{NeuN}^{+}, \mathrm{LC}^{+}, \mathrm{p}^{2} 2^{+}$, and $\mathrm{Iba}-1^{+}$cells was normalized to the total imaged area $\left(\mathrm{mm}^{2}\right)$ using the $\mathrm{NIH}$ Image $\mathrm{J}$ software $(1.43 ; \mathrm{NIH})$. Myelin images were acquired using a Leica TCS SP5 II Tunable Spectral Confocal microscope system (Leica Microsystems, Bannockburn, IL). All IHC staining experiments were performed with appropriate positive control tissue, as well as primary/secondary only negative controls.

\section{Flow cytometry and ex vivo functional assays}

Mice were perfused with $40 \mathrm{ml}$ of cold saline and the ipsilateral (i.e., craniotomy-side) hemisphere was isolated $[18,28]$. The olfactory bulb and cerebellum were removed, brains were halved along the interhemispheric fissure, and the ipsilateral hemisphere was placed separately in complete Roswell Park Memorial Institute (RPMI) 1640 (Invitrogen, Cat\# 22400105) medium and mechanically and enzymatically digested in collagenase/dispase (1 mg/ml, Roche Diagnostics, Cat\# 10269638001), 
papain (5U/ml, Worthington Biochemical, Cat\# LS003119), 0.5M EDTA (1:1000, Invitrogen, Cat\# 15575020), and DNAse I (10 mg/ml, Roche Diagnostics, Cat\# 10104159001) for $1 \mathrm{~h}$ at $37^{\circ} \mathrm{C}$ on a

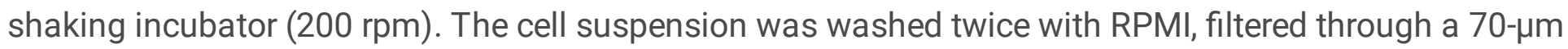
filter, and RPMI was added to a final volume of $5 \mathrm{ml} /$ hemisphere and kept on ice. Cells were then transferred into FACS tubes and washed with FACS buffer. Cells were then incubated with TruStain FcX Block (Biolegend, Cat\# 101320), for $10 \mathrm{~min}$ on ice, and stained for the following surface antigens (see Table 1): CD45-eF450 (eBioscience, Cat\# 48-0451-82), CD11b-APCeF780 (eBioscience, Cat\# 47-0112-82), Ly6C-AF700 (Biolegend, Cat\# 128024), and MHCI-PECy7 (Biolegend, Cat\# 114616). The fixable viability dye Zombie Aqua was used for live/dead discrimination (Biolegend, Cat\# 423102). Cells were then washed in FACS buffer, fixed in $2 \%$ paraformaldehyde for $10 \mathrm{~min}$, and washed once more prior to adding 500 ul FACS buffer. Intracellular staining for Ki67-PECy7 (Biolegend, Cat\# 652426), PCNA-AF647 (Biolegend, Cat\# 307912), CD68-PerCPCy5.5 (Biolegend, Cat\# 137010), NeuN-PE (Millipore Sigma, Cat\# FCMAB317PE), Vglut1-APC (StressMarq, Cat\# SMC-394D-APC), Lamp1-PerCPCy5.5 (Biolegend, Cat\# 121626), Lamp2-PE (Biolegend, Cat\# 108506), Sqstm1/p62-AF647 (Novus Biologicals, Cat\# NBP142822AF647), ATG5-AF647 (Biolegend, Cat\# 847410), ATG7-AF700 (R\&D Systems, Cat\# FAB6608N), Ubiquitin-AF647 (Biolegend, Cat\# 838710), H3-AF647 (Cell Signaling Technology, Cat\# 12230S), Acetylated (Ac) Lysine (Lys)-PECy7 (Biolegend, Cat\# 623408), H3-Ac-Lys9-AF488 (Cell Signaling Technology, Cat\# 9683S), H3-Ac-Lys18-AF488 (Cell Signaling Technology, Cat\# 73508S), H3-Ac-Lys27AF647 (Cell Signaling Technology, Cat\# 39030S), H3-Ac-Lys36-AF647 (Cell Signaling Technology, Cat\# 84061S), Phospho(ser149)-H2A.X-PECy7 (Biolegend, Cat\# 613420), p16-APC (StressMarq, Cat\# SPC1280D-APC), and p21-AF488 (RND-NBP2-43697AF488) was performed using Cytofix/Cytoperm Fixation/Permeablization Kit (BD Biosciences, Cat\# 554714) according to manufacturer's instructions and as described previously [24]. Cytokine staining for TNF-PECy7 (Biolegend, Cat\# 506324, and IL-1 $\beta-$ PerCPeF710 (eBioscience, Cat\# 46-7114-82) was performed after $3 \mathrm{~h}$ incubation with Brefeldin A (Biolegend, Cat\# 420601) at $37^{\circ} \mathrm{C}$ followed by fixation/permeabilization.

The following commercially available dyes (see Table 1) were used for ex vivo cell staining according to the manufacturer's instructions: H2dcfda (DCF, ThermoFisher, Cat\# D399), LysoTracker Deep Red (ThermoFisher, Cat\# L12492), Cyto-ID Autophagy Detection Kit (Enzo Life Sciences, Cat\# ENZ-51031K200), BODIPY 493/503 (ThermoFisher, Cat\# D3922), Lipi-Blue (Dojindo, Cat\# LD01-10), FluoroMyelin Red (ThermoFisher, Cat\# F34652), FerroOrange (Dojindo, Cat\# F374-12), ProteoStat Aggresome Detection Kit (Enzo Life Sciences, Cat\# ENZ-51035-K100), MitoSpy Red CMXRos (Biolegend, Cat\# 424802), Glucose Uptake Assay Kit (Cayman Chemical Company, Cat\# 600470), Biotracker ATP-Red (Millipore Sigma, Cat\# SCT045), BODIPY-Pepstatin A (ThermoFisher, Cat\# P12271), and LysoLive ${ }^{\text {TM }}$ Lysosomal Acid Lipase Assay Kit (Abcam, Cat\# ab253380).

Data were acquired on a BD LSRFortessa cytometer using FACSDiva 6.0 (BD Biosciences) and analyzed using FlowJo (Treestar Inc.). At least 5-10 million events were collected for each sample. Countbright Absolute Counting Beads (ThermoFisher, Cat\# C36950) were used to estimate cell counts per the manufacturer's instructions. Data were expressed as counts/hemisphere. Leukocytes were first gated using a splenocyte reference (SSC-A vs FSC-A). Singlets were gated (FSC-H vs FSC-W), and live cells were 
gated based on Zombie Aqua exclusion (SSC-A vs Zombie Aqua-Bv510). Resident microglia were identified as the $C D 45^{\text {int }} C D 11 b^{+} L y 6 C^{-}$population, whereas peripheral leukocytes were identified as $\mathrm{CD} 45^{\text {hi }} \mathrm{CD} 11 \mathrm{~b}^{+}$myeloid cells or $\mathrm{CD} 45^{\text {hi }} \mathrm{CD} 11 \mathrm{~b}^{-}$lymphocytes. Cell type-matched fluorescence minus one (FMO) controls were used to determine the positivity of each antibody and indicator dye [32].

\section{Statistical analysis}

All quantitative data are plotted as mean \pm standard error of mean. Statistical analysis was performed with Sigmaplot Version 12 (Systat software) or Graphpad Prism Version 4 for Windows (Graphpad Software, Inc). Kaplan-Meier survival curves were analyzed using the log-rank Mantel-Cox test. When comparing between two individual samples/groups, statistical significance was evaluated with the Student's unpaired t-tests (detailed in figure legends). Comparisons within each surgery group were analyzed with 2-way ANOVA group analysis followed by multiple comparisons Dunnett's or Tukey's posthoc test. For non-parametric data, the Mann Whitney test was used. A p value of $\leq 0.05$ was considered as statistically significant.

\section{Results}

\section{Old age decreases long-term survival and gait performance after TBI}

To determine whether old age impacts long-term recovery after TBI we assessed young and old male C57BL/ 6 mice using a battery of behavioral tests over the course of a 12-week period (Fig. 1a). Old mice showed significantly higher mortality beginning at 9 weeks as determined by the Mantel-Cox test (Fig. 1b). Body weights gradually increased in young mice and decreased in old mice in a normal age-related fashion, however, TBI-induced weight loss was evident early during the first week in the older group (Fig. 1c). To rule out any confounding effects in motor performance caused by differences in pain sensitivity we subjected mice to the hot plate test. Both age groups displayed similar increases in pain sensitivity to heat stimuli between 3-6 weeks after injury (Fig. 1d).

Motor function was measured using the open field (OF) for spontaneous locomotor activity, Catwalk for gait analysis, rotarod for balance and stamina, and grip testing for forelimb strength. Baseline (i.e., preTBI) motor function was significantly reduced with age in all tests. A significant group effect of injury was seen in rotarod performance, especially in young mice which showed decreased latency to fall at every timepoint after TBI (Fig. 1e). Forelimb grip strength was significantly reduced during the first week for both age groups but rebounded back to baseline or higher by 3 weeks (Fig. 1f). Together these data show that the effects of normal aging are more pronounced than TBI. Motor recovery was evident in both age groups for OF behavior and grip strength, while long-term deficits were seen in gait dynamics and rotarod performance.

OF results showed an injury effect in the total distance traveled and mean speed, marked by an early increase in each metric during the first week (Fig. 1g-i). This finding could not be attributed to increased anxiety-like behavior as no change in inner/outer zone preference was seen for either age (Fig. 1j). Gait 
analysis showed significant and lasting changes in all four limbs for all mice following injury (Fig. S1) but given the unilateral nature of the injury we focused on the contralateral, or right-side limb dynamics. Our results revealed long-term deficits in step sequence regularity and pattern for both age groups at 12 weeks post-injury (Fig. 1k, I). The duration of contact with the walkway of the right forepaw, or stand time, was chronically increased in old, but not young TBI mice (Fig. 1m). Stride length was impeded early at 3 weeks in old mice and persisted up to 12 weeks (Fig. 1n). No injury effect was seen in the swing time for the right forelimb (Fig. 10); however, swing speed was significantly increased at 12 weeks in old mice (Fig. 1p). Chronic alterations in body speed variation were evident in both age groups (Fig. 1q), whereas only young mice exhibited significantly decreased body speed late after TBI (Fig. 1r).

\section{Old age exacerbates long-term cognitive dysfunction after TBI}

To investigate the role of age on cognitive decline following TBI we examined mice using Y-maze and novel object recognition (NOR) testing, to assess short-term spatial working memory and long-term recognition memory, respectively. Significant group effects of age and injury were seen in the percentage of spontaneous alternations in the Y-maze test (Fig. 2a); however, no difference was seen between pre$\mathrm{TBI}$ and 12 weeks post-injury for either group. An injury-induced decrease in the number of arm entries was also seen in both age groups, with aged mice showing relatively fewer arm entries in general (Fig. 2b). NOR testing showed no clear preference in time spent between left and right objects during the sample phase, except for the young sham group (Fig. 2c). During the choice phase, a statistical preference was found for the novel object in all groups, except for the old TBI group which displayed late impairment in recognition memory (Fig. 2d). These findings indicate that old age is associated with a more pronounced loss of recognition rather than spatial memory during the chronic phase of TBI.

To assess depressive-like phenotype we subjected mice to novelty suppressed feeding (NSF) and social interaction testing late after TBI. Given the high degree of mortality seen in the aged TBI group, we chose tests that were less stressful than those that typically measure behavioral despair. In the NSF test, a significant effect of injury, but not age, was seen in the ratio of time required for food-deprived mice to travel to the center zone of a novel versus familiar environment to obtain food (Fig. 2e). We then evaluated social behavior in chronically injured mice. No preference in time spent between left and right cups was seen in any group (Fig. 2f). However, when one cup was replaced with a stranger mouse, all groups spent significantly more time exploring the mouse (Fig. $\mathbf{2 g}$ ). Moreover, when the other empty cup was replaced with a second stranger mouse, all groups showed a preference for the novel mouse (Fig. $2 \mathrm{~h}$ ). While an effect of age was found in time spent exploring the second stranger mouse (purple bars) in sham mice by Tukey's post-hoc test for multiple comparisons $(p<0.05)$, no statistical differences were seen between the injury groups. These data suggest that despite modest differences at baseline, depression-like behavior is prevalent in all age groups following TBI.

\section{Old age increases lesion volume, white matter loss, and microglial activation at 16 weeks following TBI}

We determined the effect of old age on chronic neurodegeneration after TBI. Quantification of cresyl violet stained tissue sections revealed significantly larger lesion volumes in old mice compared to their 
younger counterparts (Fig. 3a-b). IHC confirmed there were fewer NeuN-positive neurons in the ipsilateral cortex of old mice at 16 weeks post-injury (Fig. 3c-d). Concomitantly, the number of Iba1-positive microglia was found to be statistically increased in the old TBI cortex, but not young, as determined by two-way ANOVA with Tukey's multiple comparison's test (Fig. 3e-f). Age-related pathological

abnormalities were also seen in myelination of the medial corpus collosum as evidenced by Fluoromyelin stain intensity (Fig. $\mathbf{3 g}-\mathbf{h}$ ). Together, we demonstrate a positive association between microglia/macrophage number, severity of neuronal degeneration, and white matter degradation after chronic TBI, which is exacerbated in older mice.

\section{Gene pathways associated with microglia activation, phagocytosis, and autophagy are elevated with age in both the injured cortex and hippocampus}

To address age-related differences in the transcriptional response to TBI during the chronic phase, ipsilateral cortical and hippocampal tissues were sampled from young and old injured mice at 16 weeks post-injury. The Neuroinflammation panel tested a total of 757 genes within three themes of Immunity \& Inflammation, Neurobiology \& Neuropathology and Metabolism \& Stress [28, 29]. PCA of all normalized gene counts in the cortex and hippocampus revealed a distinct separation of samples into individual groups across the first two principal coordinates (Fig. 4a-b). In the cortex, the first component accounted for the majority of the variation (20.2\%) across samples and separated the groups by injury, while the second principal coordinate (12.9\%) separated the groups by age (Fig. 4a). To further analyze the connection between aging, TBI, and the behaviors reflective of cognitive decline and depression that we observed in tested mice, hippocampal tissue was also analyzed. In the hippocampus, injury accounted for $19.2 \%$ of the variation compared to $15.3 \%$ by age (Fig. 4 b). In both regions, the old TBI samples clustered further away on the x-axis compared to the young TBI and two sham groups, perhaps reflecting the higher number of inflammatory genes that were increased in the brain of old mice at 16 weeks postTBI.

Four pairwise comparisons of perilesional cortices and hippocampi were performed and outlined in Fig. S2: (1) young sham vs. old sham - (Set 1); (2) young TBI vs. young sham - (Set 2); (3) old TBI vs. old sham - (Set 3); and (4) old TBI vs. young TBI - (Set 4). TBI resulted in increased expression of genes in both young and old mice (Set 2 and 3), while age-related differences were seen in both baseline (Set 1) and TBI conditions (Set 4). At baseline, 130 genes (17.1\%) were differentially expressed (DE) between young and old sham groups, indicating that age affected the homeostatic regulation of genes related to the inflammatory process in a fundamental way (Fig. S2a). In the hippocampus, 121 genes were found to be DE with age (Fig. S2b). The number genes found to be down-regulated was greater in the cortex than hippocampus for both Sets 3 and 4 . Venn diagrams illustrate the number of DE genes that overlap between Sets 1, 2, 3, and 4 (Fig. S2c-d). With further analysis we were able to identify 83 genes in the cortex that were uniquely related to age and do not overlap with any genes from Set 2 and Set 3 (Fig. S2c). In addition, a total of 130 genes were found to be related to TBI injury effects (Fig. S2c). In the hippocampus, a total of 74 genes were downregulated and 47 genes were upregulated when comparing samples in Old Sham to Young Sham (Fig. S2b). At 16 weeks after injury, Set 2 had as many as 126 
genes within the inflammation panel downregulated while only 21 genes were upregulated. Similarly, the comparison between Old TBI versus Old Sham showed a total of 110 DE genes, with 83 being downregulated in the injury group while a mere 27 genes were upregulated. As demonstrated in the adjacent Venn diagrams, we identified 100 age-related genes and 101 injury-related genes (Fig. S2d).

An initial analysis of the DE genes in each set showed several genes with large fold changes in the cortex, as illustrated in a volcano plot for each gene set (Fig. $4 \mathrm{C}-\mathrm{j}$ ). To further investigate the effects of normal aging on transcriptional activation, we compared the percentage of genes modified by age (Set 1 and 4 ) for each pathway annotation. All pathways were affected with advanced age, but genes that facilitate oligodendrocyte function, neurons and neurotransmission, autophagy, astrocyte function, innate immune response, epigenetic regulation, and microglia function were far more likely to be activated in the cortex of old sham mice compared to young sham control. Alternatively, pathway analysis of genes related to injury (Set 2 and 3) showed that genes associated with oligodendrocyte function, angiogenesis, neurons and neurotransmission, matrix remodeling, astrocyte function, microglia function, and cellular stress were predominantly activated after injury (Fig. 4c-d). Pathway analysis of DE genes in the cortex is shown (Fig. 5). A heatmap of DE genes implicated in each notated pathway generally show an age-related upregulation in the chronic phase of TBI (Fig. 5a-g). The complement pathway, which has been previously implicated in age-related neurodegenerative disease and synaptic maintenance, was found to be increased both with age and injury (Fig. 5h). Genes associated with microglia function, the complement pathway, and autophagy regulation were also up-regulated with age and/or injury in the hippocampus (Fig. 6a-c). Genes associated with epigenetic regulation were also increased with age and injury (Fig. 6d). A heatmap of DE genes implicated in astrocyte function, oligodendrocyte function, and neurons \& neurotransmission showed a more mixed expression pattern after injury (Fig. 6e-g). More specifically, the age- and chronic injury-related upregulation of Axl, CD14, CD68, CD74, Ctss, Clec7a, Trem2, and Tyrobp genes in both the cortex and hippocampus is consistent with that seen in the multiple models of agerelated neurodegenerative diseases, and is representative of a disease-activated microglia (DAM) signature that reflects a pathological state of the CNS [33].

As an additional measure for examining transcriptional changes, we validated several genes that showed significant changes via real-time quantitative PCR (Fig. S3 and S4). For example, the gene Clec7a that encodes a glycoprotein linked to the modulation of the natural killer gene complex region and connected to the innate immune response functions. Real-time qPCR validation of this gene revealed significantly higher expression levels of Clec7a in the cerebral cortex of Old TBI mice compared to the Old Sham group, but barely any change in the Young TBI versus Young Sham group. Another gene, ncf1 (neutrophil cytosolic factor 1), encodes a subunit of neutrophil NADPH oxidase, which is activated to produce superoxide anions. Taken together, our results show a diverse transcriptional response of inflammatory genes in old mice compared to young mice after TBI, with several genes showing differential expression between the Old TBI and Young TBI groups.

To better illustrate the relationship between neuropathology and expression of autophagic markers, we confirmed immuno-reactivity of LC3 and p62 at the protein level in the perilesional cortex (Fig. 7a-d). 
Significant effects of age- and TBI were seen in both the number of LC3-positive and p62-positive cells at 16 weeks post-injury, consistent with our Nanostring findings. In sum, these data suggest autophagic changes in the aged and injured brain may precipitate chronic microglial activation and neuronal degeneration.

\section{Old age increases infiltration of lymphocytes and exaggerates microglial responses to TBI}

Next, we investigated the cellular inflammatory response at early (48 h) and late (16 weeks) timepoints after TBI. Brain leukocyte identification and characterization was performed using flow cytometry. Old age was associated with fewer microglia (CD45 $\left.{ }^{\text {int }} C D 11 b^{+} L y 6 C^{-}\right)$and less robust microglial accumulation in terms of absolute number (Fig. 8a-b). Normal age-related reductions in microglia number have been reported previously by our group and others and likely reflects age-related dystrophy and proliferative senescence [34]. The number of infiltrating myeloid cells (CD45 $\left.{ }^{\text {hi }} C D 11 b^{+}\right)$, however, was comparable between young and old groups and showed two-way ANOVA group effects of injury and age, including a significant interaction between them (Fig. 8c). Delayed infiltration and accumulation of putative lymphocyte populations (CD $45^{\mathrm{hi}} \mathrm{CD} 11 \mathrm{~b}$ ) was dramatically in old mice at all timepoints post-injury (Fig. 8d). These findings highlight the profound effects of aging on the central and peripheral immune response to $\mathrm{TBI}$ and suggests that blood-brain barrier integrity may be uniquely compromised in the aged brain long after injury.

To address the rapidly shifting dynamics of microglia proliferation that occurs during the first week of injury, we examined two commonly used nuclear protein markers of cellular proliferation, Ki67 and proliferating cell nuclear antigen (PCNA). Both markers were significantly upregulated in microglia at $48 \mathrm{~h}$ after TBI, but no statistical difference between injury groups was found (Fig. $\mathbf{8 e}$-f). Despite being fewer in number, microglia in old sham mice showed significantly higher basal expression of Ki67, indicating active cell cycling. The percentage of Ki67-positive microglia remained elevated late after TBI in young but not old microglia as determined by Dunnett's test $(p=0.05)$. These data do not indicate age-related difference in the kinetic progression of microglial proliferation at $48 \mathrm{~h}$, however, during the chronic stages of injury microglia from old TBI mice showed reduced proliferative capacity relative to sham compared to those from the young TBI group.

We then examined the activation state of microglia based on light scatter properties and ex vivo functional analysis. Significant effects of age and injury were seen microglia size and granularity at all timepoints, as measured by forward and side scatter intensity, respectively (Fig. S5a-b). Old microglia became markedly more granular after $48 \mathrm{~h}$ post-injury compared to young microglia, suggesting increased inflammatory activation. Reactive oxygen species (ROS) production is associated with oxidative stress which can exacerbate neurodegeneration [18, 35]. Old microglia had dramatically higher ROS production at baseline and at all timepoints post-injury, whereas young microglia exhibited a more robust increase in ROS relative to its respective sham control (Fig. $\mathbf{8 g}$ ). The percentage of microglia producing detectable ROS remained elevated at 16 weeks in young mice as determined by Dunnett's test. Lastly, we measured cytokine production in microglia which showed significant effects of both age and 
injury in expression level of IL1 $\beta$ and TNF protein (Fig. 8h-i). Both cytokines showed an age-related increase during the acute phase of TBI, which largely abated by 16 weeks $(p<0.05)$. Taken together, our data imply that old age primes microglia to become more reactive to acute $\mathrm{TBI}$, consistent with previous work. Independent of any change in cell number, basic features of microglial activation during the chronic phase of TBI appear to be more subtle on a per cell basis.

\section{Microglial phagocytosis of neurons, expression of autophagy markers, and lipofuscin content are increased with age and injury}

The emergence of a DAM signature led us to surmise that age-related deficits in autophagy function underlie chronic microglial activation and dysfunction following TBI. To better understand the temporal effects of TBI on microglia function, we performed an extensive characterization of phagocytosis and autophagy informed largely by our NanoString results indicating age-related increases in these specific pathways late after injury. Phagocytosis was measured by intracellular detection of neuronal antigens, myelin content, and the lysosomal/endosomal membrane marker CD68. Significant group effects of both age and injury were seen in the percentage of NeuN-positive microglia (Fig. 9a). The frequency of NeuNpositive microglia increased sharply at $48 \mathrm{~h}$ in both age groups and remained high at 16 weeks in old mice. Similar changes were seen in intracellular myelin content; however, while levels trended higher in old mice at 16 weeks $(p=0.06)$ it did not meet statistical significance in the two-way ANOVA multiple comparisons test (Fig. 9b). Intracellular detection of vesicular glutamate transporter 1 (Vglut1), a synaptic protein in neurons, was also increased acutely in microglia after TBI (Fig. 9c). A group effect of age was found, indicating a higher presence of this synaptic marker in old microglia. The phagocytosis marker CD68 was significantly higher in old microglia both at baseline and at 16 weeks but was elevated to a similar extent at $48 \mathrm{~h}$ in both age groups (Fig. 9d). These findings suggest that microglial phagocytosis of dead or dying neurons is robust in the early stages of $\mathrm{TBI}$, and modestly increased with both age and time post-injury, consistent with our gene expression data.

Lysosome and autophagosome content in microglia were significantly increased with both age and injury and remained elevated for up to 16 weeks (Fig. 9eg). Protein expression of Sqstm1/p62 and Lamp2 were increased at $48 \mathrm{~h}$, but no effect of age was seen (Fig. 9h-i). The ubiquitin-proteasome system degrades intracellular proteins into peptide fragments that can be presented by major histocompatibility complex (MHC) class I molecules. Significant group effects of age and injury were found in surface expression of $\mathrm{MHCl}$ with the highest expression seen at $48 \mathrm{~h}$ (Fig. 9j). Defects in the autophagy pathway can result in the cytosolic and lysosomal accumulation of an autofluorescent amalgam of oxidized lipids, proteins, and metals known as lipofuscin. Group effects of age and injury were seen in intracellular lipid content, iron levels, peptide/protein aggregation, and autofluorescence (Fig. S5c-f). Neutral lipid and iron content increased sharply at $48 \mathrm{~h}$, consistent with the increase in phagocytic activity. Protein aggregation and autofluorescence were largely unperturbed at $48 \mathrm{~h}$, however, both were found to be elevated above sham levels at 16 weeks in young microglia as determined by Dunnett's test ( $p<0.05$ and $p<0.05$, respectively). Collectively, these data imply that phagocytosis and autophagic function are exacerbated with age and $\mathrm{TBI}$, consistent with our DAM gene signature. Although these processes are more active during the acute 
stages of injury, many markers, including the accumulation of lipofuscin material, show a sustained increase during the chronic phase.

\section{Age-related changes in microglial histone acetylation patterns, metabolic activity levels, and expression of senescence markers are altered after TBI}

Given the widespread effects of aging on microglial function, we also evaluated changes in epigenetic regulation, metabolic activity, and senescence-like phenotype following TBI. Microglia activation is associated with increased histone deacetylase (HDAC) and sirtuin (SIRT) activity resulting in hypoacetylation of histones and non-histone proteins and increased expression of inflammatory markers [36]. Our results show an age-related loss of histone $3(\mathrm{H} 3)$ within the general microglia population, however, $\mathrm{H} 3$ expression levels were dramatically increased at $48 \mathrm{~h}$ in both age groups (Fig. 10a). The percentage of microglia expressing acetylated lysine residues was significantly reduced with age and injury indirectly suggesting increased HDAC and SIRT activity (Fig. 10b). To further probe the impact of TBI on histone modification we examined acetylation patterns on H3. Significant effects of age and injury, or injury alone, were seen in $\mathrm{H} 3$ hypoacetylation of the lysine residue at $\mathrm{N}$-terminal positions 9,18 , 27 , and 36 (Fig. 10c-f). These data suggest that aging and TBI result in the removal of acetyl groups from $\mathrm{H} 3$ which is known to increase chromatin condensation and decrease gene transcription.

TBI acutely increased mitochondrial activity, glucose uptake, and ATP production in microglia (Fig. 11aC). At 16 weeks post-injury, the cellular demand for glucose remained higher than sham control in young mice as determined by Dunnett's test $(p<0.01)$. These findings suggest that TBI causes a metabolic crisis in microglia that is associated with a chronic increase in glucose uptake in young mice.

We have previously demonstrated that a small population of microglia upregulate markers of cellular senescence in an age- and TBI-dependent manner [37]. However, the sustained presence of a senescentlike phenotype has yet to be reported in microglia at chronic timepoints after injury. Expression of the DNA damage sensor, p-H2AX, showed a basal elevation with age (Fig. 11d). Interestingly, p-H2AX levels were significantly decreased at $48 \mathrm{~h}$, likely due to a stress-mediated increase in DNA repair mechanisms. Although expression was further increased from $48 \mathrm{~h}$ to 16 weeks, levels did not surpass that seen in sham controls. Expression of the cell cycle arrest markers p16 and p21 were increased with normal aging (Fig. 11ef). The percent of p16-positive microglia trended higher in young TBI mice at 16 weeks compared to sham, but significance was not achieved by two-way ANOVA multiple comparison test. It should be noted that the absolute number of p16-positive microglia may be significantly increased in the absence of a statistical change in percentage. In contrast, a robust increase in the relative expression of p21 was seen at $48 \mathrm{~h}$ in both age groups, however, levels were higher in old microglia at both timepoints post-injury (Fig. 11f). Together these results indicate that aging interacts with TBI to modify key aspects of microglial homeostasis, including post-translational epigenetic regulation, metabolic function, and development of a senescence-like phenotype.

\section{Trehalose treatment enhances functional recovery in motor and cognitive tasks and decreases depressive-like behavior}


Old mice were administered trehalose or sucrose control in their drinking water beginning on $\mathrm{d} 1 \mathrm{for}$ up to 8 weeks after surgery. A battery of behavioral tests was performed to monitor changes in motor performance, cognitive function, and anxiety/depression (Fig. 12a). Trehalose treatment resulted in a delayed decrease in body weight (Fig. 12b). No statistical deficits were seen in rotarod performance at any timepoint in mice treated with trehalose, whereas mice treated with sucrose control exhibited lasting impairment for up to 8 weeks after TBI (Fig. 12C). OF activity was then assessed (Fig. 12d). Interestingly, sucrose-treated mice traveled significant shorter distances in the OF during the first week of injury (Fig.

12e). This was also accompanied by a decrease in speed, not seen in trehalose-treated mice (Fig. 12f). A statistical increase in time spent in the center zone of the OF late after injury was recorded in mice administered trehalose, suggesting that enhancing autophagy during TBI may result in delayed anxiolytic effects (Fig. 12g). These data suggest continued administration of trehalose may attenuate motor deficits in aged mice after TBI.

To address the effect of trehalose on long-term cognitive outcomes in old mice, we evaluated mice using the Y-maze and NOR tests. While both treatment groups showed a temporary reduction in the number of arm entries early after TBI, no therapeutic effects were seen (Fig. 12h-i). However, trehalose-treated mice did show improvement in recognition memory at 8 weeks post-TBI compared to sucrose control (Fig. 12jk). Lastly, NSF and social interaction tests were employed to evaluate anxiety/depressive-like phenotype. Trehalose-treated mice displayed shorter latency times to reach food at the center of the OF compared to sucrose control, suggesting less depression-related behavior, consistent with our OF findings (Fig. 12I). Finally, social interaction testing showed that trehalose increased the amount of time spent interacting with a novel stranger mouse in the social discrimination phase (i.e., sequence 3 ) of the test (Fig. 12m-o). Together these findings show that continued trehalose treatment can preserve recognition memory and reduce anxiety/depression in old mice late after injury.

\section{Trehalose treatment reduces chronic brain inflammation and phagocytosis of neuronal synapses by modulating multiple steps of the autophagy pathway in microglia}

To better understand the impact of trehalose on chronic microglia activation and neuroinflammation in old mice after TBI we examined autophagic function at 9 weeks post-injury. Trehalose treatment caused a clear reduction in the number of proliferated microglia (CD45 $\left.{ }^{\text {int }} \mathrm{CD} 11 \mathrm{~b}^{+} \mathrm{Ly} 6 \mathrm{C}^{-}\right)$present in the ipsilateral hemisphere and significantly prevented the delayed recruitment or accumulation of peripherally-derived

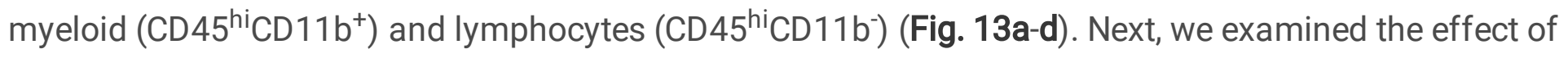
trehalose on phagocytosis. A significant injury-related increase was found in microglial expression of CD68, but no effect of treatment was seen (Fig. 13e). Lipid content trended higher in sucrose-treated mice $(p=0.05)$, while trehalose treatment caused no change (Fig. 13f). The percentage of microglia that engulfed neuronal Vglut 1 and the relative level of intracellular NeuN protein were both significantly lower in trehalose-treated mice (Fig. 13g-h).

A comprehensive analysis of autophagy-related markers showed no effect of treatment or injury at 8 weeks as measured by LysoTracker dye, lamp1, lamp2, and Sqstm1/p62 (Fig. S6a-d). Although increased 
formation of LC3Il-positive autophagosomes was found in sucrose- but not trehalose-treated mice, this difference was not statistically significant ( $p=0.05$; Fig. 14a). A group effect of TBI was seen in ATG5 and ATG7 expression in microglia; trehalose treatment prevented a significant reduction in ATG7 (Fig. 14b-c). Examination of lysosomal enzymatic activity in microglia revealed a robust increase in cathepsin $D$ and lysosomal lipase activity in mice treated with trehalose (Fig. 14d-e). There was a trend towards increased ubiquitin expression in microglia in sucrose-treated mice, but changes did not reach significance $(p=0.05$; Fig. 14f). Whereas antigen presentation by $\mathrm{MHCl}$ was more augmented after trehalose treatment, it did not alter injury-induced increases in intracellular protein aggregation (Fig. 14g-h). Lastly, trehalosemediated protection was associated with attenuated lysine acetylation in microglia (Fig. S6e). Taken together, our results indicate that long-term trehalose treatment reduces chronic brain inflammation via modulation of several key proteins involved in the autophagy pathway, including the upstream regulation of phagocytosis.

\section{Discussion}

We show that old age augments the expression of the disease-associated microglia signature during the chronic phase of TBI. This transcriptional and functional response is characterized by the downregulation of homeostatic pathways and upregulation of genes involved in lysosomal and phagocytic pathways. Neuronal/myelin engulfment, autophagic processes, and inflammatory activation were exacerbated with advanced age in microglia at both acute and chronic times after injury. Continuous treatment with orally administered trehalose following brain injury improved long-term neurological recovery in old mice, which was associated with chronically reduced phagocytic activity and increased lysosomal function. Our findings suggest that age-related dysregulation of autophagy plays a role in chronic microglial activation, neuroinflammation, and recovery following brain injury.

An extensive longitudinal assessment of functional recovery in aged mice following experimental TBI showed that older mice have similar recovery patterns for many, but not all behavioral tests compared to younger mice. Indeed, overall performance was more profoundly affected by normal aging than TBI. Thus, it can be problematic to differentiate effects of aging from those of injury due to differences in baseline performance. Thus, we have expressed the behavioral data as absolute changes rather than as percent changes from baseline to better illustrate recovery dynamics within groups. One conclusion from our findings is that older mice show some behavioral restoration late after TBl; thus, the same reparative processes that may be therapeutically modulated in young mice may also be targeted in older animals.

Using the NanoString Neuroinflammation Panel, TBI caused transcriptional activation of more than 100 additional inflammation-related genes in the cortex, and 40 fewer genes in the hippocampus of injured aged mice versus sham control as compared to changes in the injured young group. Based on the incremental increases in expression of DAM genes observed with both aging and chronic injury, these findings are consistent with the conclusion that TBI accelerates normal age-related expression of this inflammatory signature which may contribute to accelerated neurological impairment. Moreover, this work suggests that many prognostic biomarkers of TBI outcome, including the activation of specific gene 
networks, may need to be reassessed in older patients, given the normal age-related increases in gene expression levels and protein concentrations.

Age and injury-related increases in the expression of genes associated with the complement pathway is consistent with increased innate immune activation and supports a role for phagocytosis in the chronic brain injury setting. Complement activation products opsonize synaptic material on neurons for phagocytic removal, which during development, functions to prune excess synapses to refine neural circuitry [38]. This process is also important for synaptic plasticity throughout life. However, an increasing number of studies have recently demonstrated that excessive complement-mediated synaptic engulfment is a pathological process in the context of neurodegenerative disease, including TBI [39]. This would be consistent with our ex vivo phagocytosis data showing increased microglial engulfment of neuronal/synaptic antigens and decreased numbers of viable neurons and synaptic plasticity late after TBI [17]. Genes associated with astrocyte function were also relatively more upregulated in the aged brain late after TBI. Astrocytes can induce the expression of C1q in neurons via TGF- $\beta$ [40]. C1q is involved in the pathological pathway of several neurodegenerative diseases due to its role in abnormal protein aggregate clearance, astrocyte reactivation, binding and activation of microglia, or inflammatory responses [41]. It remains to be seen whether complement gene expression is elevated in astrocytes during the chronic phase of $\mathrm{TBI}$, and if $\mathrm{C} 1 \mathrm{q}, \mathrm{C} 3$, and $\mathrm{C} 4 \mathrm{a}$ factors are localized at synapses and neurons in affected brain regions.

Brain aging leads to decline in autophagy efficiency, accumulation of lipofuscin, and increases in inflammatory activity including ROS production. Moreover, previous work has found that cellular and molecular markers of inflammation in the brain are acutely altered with age after TBI $[7,8,37,42]$. The present study has now captured similar changes in aged mice during the chronic phase of TBI using a combination of ex vivo cellular assays, transcriptional gene profiling, and histological approaches. At the cellular level, the acute phase of injury was marked by an increase in infiltrating myeloid cells and microglial proliferation, whereas the chronic phase is highlighted by delayed lymphocyte recruitment. The leukocyte dynamics, however, were altered with age. Older mice showed significantly fewer microglia in general, either due cell senescence and/or death or the cell isolation procedure. Nonetheless, similar changes have been seen in other CNS cell types with age, including microglia [43]. Lymphocyte infiltration was exacerbated in old mice at all timepoints. Similar changes have been reported in aged models of ischemic stroke $[44,45]$, suggesting this may be a conserved age-related hallmark of brain injury. However, lymphocyte numbers were far exceeded by the resident microglial population. Although we made efforts to characterize the age-related temporal kinetics of microglial proliferation after TBI, two timepoints alone cannot accurately capture these dynamics, especially during the fast-evolving acute period. For example, in a previous study we noted relatively greater microglial proliferation at $72 \mathrm{~h}$ postinjury in aged mice compared to young controls [37]. The fact that we did not observe this response at $48 \mathrm{~h}$ post-injury in our study might suggest that microglial proliferation is delayed with age. This would be in line with other reports, and our own data showing similar or higher expression of cell proliferation markers at that point in time for old microglia [37]. Although numbers of brain-infiltrating myeloid cells were similar between groups at $48 \mathrm{~h}$, we had previously reported that the composition of these cells 
skewed more neutrophilic in older mice [37]. Thus, the cellular immune dynamics of the neuroinflammatory response to TBI are different with older animals. Changes in leukocyte number and composition, notwithstanding the effects of aging alone, suggest the quality and severity of immune function and inflammatory activity may be markedly different in older mice.

To ascertain whether microglial reactivity at acute and chronic stages of TBI was affected in old age we performed an extensive profiling of cellular functions. In most measures, pro-inflammatory activity was relatively greater in older microglia after acute TBI. Of note, however, were the significant effects of age on phagocytosis, autophagy, and lipofuscin accumulation in microglia following TBI. In general, older microglia were more likely to engulf neurons and/or neuronal debris than their younger counterparts. Although the effects of TBI appear subtle at 16 weeks due to scaling and higher statistical stringency required for multiple comparison's testing, we have previously reported a chronic increase in phagocytosis of apoptotic neurons and myelin debris as late as 8 months post-TBI [17]. The acute phase was also characterized by a robust age-related increase in lysosome and autophagosome formation. Lipofuscin accumulation was elevated in both age groups after injury. Interestingly, intracellular protein aggregation was reduced at $48 \mathrm{~h}$ in microglia; however, by 16 weeks post-injury protein aggregation recrudesced in both age groups, implying late impairment of autophagic processes in microglia. These age-related alterations in phagocytosis, autophagy, and inflammatory activity are consistent with the agerelated increase in the DAM gene signature, and its potential role in microglial activation changes during chronic brain injury.

We also identified novel age- and TBI-driven changes in epigenetic, metabolic, and senescence markers. The microglial activation, which often persists chronically following more severe head injury is consistent with an enduring change in cellular reprogramming mediated by epigenetic mechanisms, as we have suggested previously [46]. Earlier studies have reported imbalances in epigenetic marks of DNA methylation and histone acetylation after TBI [47]. Specifically, both acetylation and methylation of histones are decreased in the brain after $\mathrm{CCl}$ injury in rodents [48-51]. TBI-induced histone acetylation is caused in part by increases in HDAC activity [52]. The functional implications of histone hypoacetylation are consistent with the neuroprotective effects shown with HDAC inhibition [50,53]. Microglia-specific deletion of HDAC1 and HDAC2 have been shown to alter development, homeostasis, and activation [54]. Thus, it would appear histone deacetylation is a pathological feature of TBI and potential driver of microglial-mediated inflammation. The present study is among the first to examine these changes at the cellular level in microglia. We found that TBI not only reduced global lysine acetylation levels, but also histone acetylation. Moreover, these changes were lasting, exacerbated with age, and reversed with trehalose treatment. Thus, our data are consistent with the view that epigenetic changes of histones occur early after TBI and are sustained in microglia. Microglia adopted a senescence phenotype as a function of age and injury. The interaction between age and injury found in our study highlights a potential mechanism for the exaggerated inflammation and neurological dysfunction seen in older animals [55-58]. 
Using aged animals, we employed a highly studied neuroprotectant and autophagic inducer as a potential therapeutic agent. Trehalose is currently in clinical trials for $A D$ and neuronal ceroidlipofuscinoses. Importantly, it is well-tolerated, widely available, low-cost and can be orally administered without additives [59]. To assess longitudinal recovery in aged animals treated with trehalose, we performed a similar behavioral battery but with shorter injury duration to minimize long-term mortality. Sucrose was used as the control group because it shares a similar molecular structure with trehalose. Like many control solutions, sucrose is not an inert substance and may exert its own effects on metabolism and neurological recovery which have not been taken into consideration here. Nevertheless, trehalose was found to be more protective in aged mice after TBI. Previous work has shown that young mice treated with trehalose perform better in Morris water maze, OF testing, and Y-maze [20]. Furthermore, trehalose is known to also act as a chemical chaperone [60] and may therefore have protective actions that are independent of autophagy. For example it has been reported that trehalose treatment is associated with upregulation of synaptophysin, doublecortin, and BDNF [20].

Given its ability to stabilize biomolecules and increase autophagy, trehalose has received growing attention as a potential neuroprotective agent $[59,61]$. However, the precise mechanisms through which trehalose acts on the autophagy pathway in microglia have not been explored. Most importantly, we found that trehalose reduces overall numbers of inflammatory immune cells in the injured brain. Whether trehalose acts directly on the brain or in the periphery to achieve this effect is not clear. Our results also showed unexpected changes in phagocytic activity late after TBI. Trehalose reduced continued phagocytosis of neurons and neuronal synapses, and attenuated lipid accumulation. This suggests that trehalose can act upstream of the autophagy pathway to potentially prevent the ingestion and accumulation of phagocytized material. Perhaps surprisingly, we did not see changes in lysosome formation or Sqstm1/p62 expression in microglia following trehalose treatment. Rather, the effects of trehalose on autophagy are likely more relevant during the acute phase of injury when phagocytosis and lysosomal degradation processes are sharply elevated [62]. Nonetheless, chronic increases in lysosomal enzyme activity were seen in trehalose-treated mice indicative of improved autophagy function. Further, trehalose prevented injury-induced increases in autophagosome formation and decreases in ATG7 expression late after TBI. Taken together, we identified several potential steps in the autophagy pathway where trehalose may exert important effects on microglia that are associated with better functional outcome following TBI

\section{Conclusion}

Our histological, cellular, and molecular findings provided complementary evidence for age-related, chronic dysregulation of microglial function and autophagic processes. We show that therapeutically modulating autophagy using trehalose can reduce chronic brain inflammation in aged mice and enhance long term recovery.

\section{Abbreviations}


AD: Alzheimer's disease;

CW: catwalk test;

CCl: controlled cortical impact;

CNS: central nervous system;

DAM: disease-associated microglia;

DE: differentially expressed;

GS: grip strength test;

HP: hot plate test;

IHC: immunohistochemistry;

MS: multiple sclerosis;

NOR: novel object recognition test;

NSF: novel suppressed feeding test;

OF: open field test;

PCA: principal component analysis;

ROS: reactive oxygen species;

SR: social recognition test;

TBI: Traumatic brain injury;

YM: Y-maze test

\section{Declarations}

\section{Ethics Approval}

All animal procedures were conducted in accordance with the guidelines set forth by the National Institutes of Health and the University of Maryland, Baltimore Institutional Animal Care and Use Committee, who approved the study protocol.

\section{Consent for publication}

Not applicable 


\section{Competing Interests}

The authors declare no conflict of interest.

Availability of data

All data generated or analyzed during this study are included in this article.

\section{Funding}

The work was supported by the National Institutes of Health Grants K99 NS116032 (RMR), RF1 NS110637 (JW), R01 NS094527 (JW), R01 NS110825 (JW), and R01 NS110635 (AIF/JW).

Author information

RMR and JW conceived the idea, designed research, and wrote the paper. RMR performed the flow cytometry and behavioral experiments, analyzed the data, and prepared the figures. YL performed behavioral experiments, the NanoString analyses, and histology analysis, prepared figures, and wrote the paper. JC performed behavioral experiments. JH performed the CCl surgeries and qPCR analysis. ZL and $\mathrm{HC}$ performed the immunohistology experiments and analyzed the data. NK, SA, YL, and JC performed flow cytometry experiments. HL performed animal perfusion, brain sectioning, histology, and assisted flow cytometry. MML and AIF edited the manuscript. All authors read and approved the final manuscript.

\section{References}

1. Brett BL, Gardner RC, Godbout J, Dams-O'Connor K, Keene CD: Traumatic Brain Injury and Risk of Neurodegenerative Disorder.Biol Psychiatry 2021.

2. Hammond FM, Corrigan JD, Ketchum JM, Malec JF, Dams-O'Connor K, Hart T, Novack TA, Bogner J, Dahdah MN, Whiteneck GG: Prevalence of Medical and Psychiatric Comorbidities Following Traumatic Brain Injury.J Head Trauma Rehabil 2019, 34:E1-E10.

3. Gan S, Shi W, Wang S, Sun Y, Yin B, Bai G, Jia X, Sun C, Niu X, Wang Z, et al: Accelerated Brain Aging in Mild Traumatic Brain Injury: Longitudinal Pattern Recognition with White Matter Integrity.J Neurotrauma 2021.

4. Gardner RC, Burke JF, Nettiksimmons J, Goldman S, Tanner CM, Yaffe K: Traumatic brain injury in later life increases risk for Parkinson disease.Ann Neuro/ 2015, 77:987-995.

5. Gardner RC, Burke JF, Nettiksimmons J, Kaup A, Barnes DE, Yaffe K: Dementia risk after traumatic brain injury vs nonbrain trauma: the role of age and severity.JAMA Neurol 2014, 71:1490-1497.

6. Gardner RC, Dams-O'Connor K, Morrissey MR, Manley GT: Geriatric Traumatic Brain Injury: Epidemiology, Outcomes, Knowledge Gaps, and Future Directions.J Neurotrauma 2018, 35:889-906.

7. Morganti JM, Riparip LK, Chou A, Liu S, Gupta N, Rosi S: Age exacerbates the CCR2/5-mediated neuroinflammatory response to traumatic brain injury.J Neuroinflammation 2016, 13:80. 
8. Krukowski K, Chou A, Feng X, Tiret B, Paladini MS, Riparip LK, Chaumeil MM, Lemere C, Rosi S: Traumatic Brain Injury in Aged Mice Induces Chronic Microglia Activation, Synapse Loss, and Complement-Dependent Memory Deficits.Int J Mol Sci 2018, 19.

9. Quan X, Song L, Zheng X, Liu S, Ding H, Li S, Xu G, Li X, Liu L: Reduction of Autophagosome Overload Attenuates Neuronal Cell Death After Traumatic Brain Injury.Neuroscience 2021, 460:107-119.

10. Lauro C, Limatola C: Metabolic Reprograming of Microglia in the Regulation of the Innate Inflammatory Response.Front Immunol 2020, 11:493.

11. Finkbeiner S: The Autophagy Lysosomal Pathway and Neurodegeneration.Cold Spring Harb Perspect Biol 2020, 12.

12. Cho KS, Lee JH, Cho J, Cha GH, Song GJ: Autophagy Modulators and Neuroinflammation.Curr Med Chem 2020, 27:955-982.

13. Wu J, Lipinski MM: Autophagy in Neurotrauma: Good, Bad, or Dysregulated.Cells 2019, 8.

14. Loeffler DA: Influence of Normal Aging on Brain Autophagy: A Complex Scenario.Front Aging Neurosci 2019, 11:49.

15. Sun M, McDonald SJ, Brady RD, Collins-Praino L, Yamakawa GR, Monif M, O'Brien TJ, Cloud GC, Sobey CG, Mychasiuk $R$, et al: The need to incorporate aged animals into the preclinical modeling of neurological conditions. Neurosci Biobehav Rev 2020, 109:114-128.

16. Iboaya A, Harris JL, Arickx AN, Nudo RJ: Models of Traumatic Brain Injury in Aged Animals: A Clinical Perspective.Neurorehabil Neural Repair 2019, 33:975-988.

17. Ritzel RM, Li Y, He J, Khan N, Doran SJ, Faden Al, Wu J: Sustained neuronal and microglial alterations are associated with diverse neurobehavioral dysfunction long after experimental brain injury.Neurobiol Dis 2020, 136:104713.

18. Ritzel RM, He J, Li Y, Cao T, Khan N, Shim B, Sabirzhanov B, Aubrecht T, Stoica BA, Faden Al, et al: Proton extrusion during oxidative burst in microglia exacerbates pathological acidosis following traumatic brain injury. Glia 2021, 69:746-764.

19. Portbury SD, Hare DJ, Sgambelloni C, Perronnes K, Portbury AJ, Finkelstein DI, Adlard PA: Trehalose Improves Cognition in the Transgenic Tg2576 Mouse Model of Alzheimer's Disease.J Alzheimers Dis 2017, 60:549-560.

20. Portbury SD, Hare DJ, Finkelstein DI, Adlard PA: Trehalose improves traumatic brain injury-induced cognitive impairment.PLoS One 2017, 12:e0183683.

21. Xu C, Li X, Wang F, Weng H, Yang P: Trehalose prevents neural tube defects by correcting maternal diabetes-suppressed autophagy and neurogenesis.Am J Physiol Endocrinol Metab 2013, 305:E667678.

22. Wu J, Zhao Z, Kumar A, Lipinski MM, Loane DJ, Stoica BA, Faden Al: Endoplasmic Reticulum Stress and Disrupted Neurogenesis in the Brain Are Associated with Cognitive Impairment and DepressiveLike Behavior after Spinal Cord Injury.J Neurotrauma 2016, 33:1919-1935. 
23. Matyas JJ, O'Driscoll CM, Yu L, Coll-Miro M, Daugherty S, Renn CL, Faden Al, Dorsey SG, Wu J: Truncated TrkB.T1-Mediated Astrocyte Dysfunction Contributes to Impaired Motor Function and Neuropathic Pain after Spinal Cord Injury.J Neurosci 2017, 37:3956-3971.

24. Doran SJ, Ritzel RM, Glaser EP, Henry RJ, Faden Al, Loane DJ: Sex Differences in Acute Neuroinflammation after Experimental Traumatic Brain Injury Are Mediated by Infiltrating Myeloid Cells.J Neurotrauma 2019, 36:1040-1053.

25. Jacotte-Simancas A, Costa-Miserachs D, Coll-Andreu M, Torras-Garcia M, Borlongan CV, Portell-Cortes I: Effects of voluntary physical exercise, citicoline, and combined treatment on object recognition memory, neurogenesis, and neuroprotection after traumatic brain injury in rats.J Neurotrauma 2015, 32:739-751.

26. Moy SS, Nadler JJ, Perez A, Barbaro RP, Johns JM, Magnuson TR, Piven J, Crawley JN: Sociability and preference for social novelty in five inbred strains: an approach to assess autistic-like behavior in mice.Genes Brain Behav 2004, 3:287-302.

27. Wu J, Zhao Z, Zhu X, Renn CL, Dorsey SG, Faden Al: Cell cycle inhibition limits development and maintenance of neuropathic pain following spinal cord injury.Pain 2016, 157:488-503.

28. Li Y, Ritzel RM, Khan N, Cao T, He J, Lei Z, Matyas JJ, Sabirzhanov B, Liu S, Li H, et al: Delayed microglial depletion after spinal cord injury reduces chronic inflammation and neurodegeneration in the brain and improves neurological recovery in male mice. Theranostics 2020, 10:11376-11403.

29. Li Y, Ritzel RM, He J, Cao T, Sabirzhanov B, Li H, Liu S, Wu LJ, Wu J: The voltage-gated proton channel Hv1 plays a detrimental role in contusion spinal cord injury via extracellular acidosismediated neuroinflammation. Brain Behav Immun 2021, 91:267-283.

30. Li Y, Jones JW, H MCC, Sarkar C, Kane MA, Koh EY, Lipinski MM, Wu J: cPLA2 activation contributes to lysosomal defects leading to impairment of autophagy after spinal cord injury. Cell Death Dis 2019, 10:531.

31. Liu S, Li Y, Choi HMC, Sarkar C, Koh EY, Wu J, Lipinski MM: Lysosomal damage after spinal cord injury causes accumulation of RIPK1 and RIPK3 proteins and potentiation of necroptosis. Cell Death Dis 2018, 9:476.

32. Ritzel RM, Al Mamun A, Crapser J, Verma R, Patel AR, Knight BE, Harris N, Mancini N, Roy-O'Reilly M, Ganesh BP, et al: CD200-CD200R1 inhibitory signaling prevents spontaneous bacterial infection and promotes resolution of neuroinflammation and recovery after stroke.J Neuroinflammation 2019, 16:40.

33. Keren-Shaul H, Spinrad A, Weiner A, Matcovitch-Natan O, Dvir-Szternfeld R, Ulland TK, David E, Baruch K, Lara-Astaiso D, Toth B, et al: A Unique Microglia Type Associated with Restricting Development of Alzheimer's Disease.Cell 2017, 169:1276-1290 e1217.

34. Zoller T, Attaai A, Potru PS, Russ T, Spittau B: Aged Mouse Cortical Microglia Display an Activation Profile Suggesting Immunotolerogenic Functions.Int J Mol Sci 2018, 19.

35. Kumar A, Barrett JP, Alvarez-Croda DM, Stoica BA, Faden Al, Loane DJ: NOX2 drives M1-like microglial/macrophage activation and neurodegeneration following experimental traumatic brain 
injury.Brain Behav Immun 2016, 58:291-309.

36. Penas C, Navarro X: Epigenetic Modifications Associated to Neuroinflammation and Neuropathic Pain After Neural Trauma.Front Cell Neurosci 2018, 12:158.

37. Ritzel RM, Doran SJ, Glaser EP, Meadows VE, Faden Al, Stoica BA, Loane DJ: Old age increases microglial senescence, exacerbates secondary neuroinflammation, and worsens neurological outcomes after acute traumatic brain injury in mice.Neurobiol Aging 2019, 77:194-206.

38. Presumey J, Bialas AR, Carroll MC: Complement System in Neural Synapse Elimination in Development and Disease.Adv Immunol 2017, 135:53-79.

39. Hammad A, Westacott L, Zaben M: The role of the complement system in traumatic brain injury: a review.J Neuroinflammation 2018, 15:24.

40. Chung WS, Allen NJ, Eroglu C: Astrocytes Control Synapse Formation, Function, and Elimination.Cold Spring Harb Perspect Biol 2015, 7:a020370.

41. Cho K: Emerging Roles of Complement Protein C1q in Neurodegeneration.Aging Dis 2019, 10:652663.

42. Chou A, Krukowski K, Morganti JM, Riparip LK, Rosi S: Persistent Infiltration and Impaired Response of Peripherally-Derived Monocytes after Traumatic Brain Injury in the Aged Brain.Int J Mol Sci 2018, 19.

43. Peters R: Ageing and the brain.Postgrad Med J 2006, 82:84-88.

44. Harris NM, Roy-O'Reilly M, Ritzel RM, Holmes A, Sansing LH, O'Keefe LM, McCullough LD, Chauhan A: Depletion of CD4 T cells provides therapeutic benefits in aged mice after ischemic stroke.Exp Neurol 2020, 326:113202.

45. Crapser J, Ritzel R, Verma R, Venna VR, Liu F, Chauhan A, Koellhoffer E, Patel A, Ricker A, Maas K, et al: Ischemic stroke induces gut permeability and enhances bacterial translocation leading to sepsis in aged mice.Aging (Albany NY) 2016, 8:1049-1063.

46. Henry RJ, Ritzel RM, Barrett JP, Doran SJ, Jiao Y, Leach JB, Szeto GL, Wu J, Stoica BA, Faden Al, Loane DJ: Microglial Depletion with CSF1R Inhibitor During Chronic Phase of Experimental Traumatic Brain Injury Reduces Neurodegeneration and Neurological Deficits.J Neurosci 2020, 40:2960-2974.

47. Nagalakshmi B, Sagarkar S, Sakharkar AJ: Epigenetic Mechanisms of Traumatic Brain Injuries.Prog Mol Biol Trans/ Sci 2018, 157:263-298.

48. Balasubramanian N, Sagarkar S, Jadhav M, Shahi N, Sirmaur R, Sakharkar AJ: Role for histone deacetylation in traumatic brain injury-induced deficits in neuropeptide $\mathrm{Y}$ in arcuate nucleus: Possible implications in feeding behaviour.Neuroendocrinology 2020.

49. Sagarkar S, Balasubramanian N, Mishra S, Choudhary AG, Kokare DM, Sakharkar AJ: Repeated mild traumatic brain injury causes persistent changes in histone deacetylase function in hippocampus: Implications in learning and memory deficits in rats.Brain Res 2019, 1711:183-192. 
50. Zhang B, West EJ, Van KC, Gurkoff GG, Zhou J, Zhang XM, Kozikowski AP, Lyeth BG: HDAC inhibitor increases histone $\mathrm{H} 3$ acetylation and reduces microglia inflammatory response following traumatic brain injury in rats. Brain Res 2008, 1226:181-191.

51. Gao WM, Chadha MS, Kline AE, Clark RS, Kochanek PM, Dixon CE, Jenkins LW: Immunohistochemical analysis of histone $\mathrm{H} 3$ acetylation and methylation-evidence for altered epigenetic signaling following traumatic brain injury in immature rats.Brain Res 2006, 1070:31-34.

52. Gibson CL, Murphy SP: Benefits of histone deacetylase inhibitors for acute brain injury: a systematic review of animal studies.J Neurochem 2010, 115:806-813.

53. Sada N, Fujita Y, Mizuta N, Ueno M, Furukawa T, Yamashita T: Inhibition of HDAC increases BDNF expression and promotes neuronal rewiring and functional recovery after brain injury.Cell Death Dis 2020, 11:655.

54. Datta M, Staszewski O, Raschi E, Frosch M, Hagemeyer N, Tay TL, Blank T, Kreutzfeldt M, Merkler D, Ziegler-Waldkirch S, et al: Histone Deacetylases 1 and 2 Regulate Microglia Function during Development, Homeostasis, and Neurodegeneration in a Context-Dependent Manner.Immunity 2018, 48:514-529 e516.

55. Schwab N, Ju Y, Hazrati LN: Early onset senescence and cognitive impairment in a murine model of repeated mTBI.Acta Neuropathol Commun 2021, 9:82.

56. Arun P, Rossetti F, Wilder DM, Sajja S, Van Albert SA, Wang Y, Gist ID, Long JB: Blast Exposure Leads to Accelerated Cellular Senescence in the Rat Brain.Front Neurol 2020, 11:438.

57. Schwab N, Grenier K, Hazrati LN: DNA repair deficiency and senescence in concussed professional athletes involved in contact sports.Acta Neuropathol Commun 2019, 7:182.

58. Tominaga T, Shimada R, Okada Y, Kawamata T, Kibayashi K: Senescence-associated-betagalactosidase staining following traumatic brain injury in the mouse cerebrum.PLoS One 2019, 14:e0213673.

59. Khalifeh M, Read MI, Barreto GE, Sahebkar A: Trehalose against Alzheimer's Disease: Insights into a Potential Therapy.Bioessays 2020, 42:e1900195.

60. Sarkar S, Davies JE, Huang Z, Tunnacliffe A, Rubinsztein DC: Trehalose, a novel mTOR-independent autophagy enhancer, accelerates the clearance of mutant huntingtin and alpha-synuclein.J Biol Chem 2007, 282:5641-5652.

61. Emanuele E: Can trehalose prevent neurodegeneration? Insights from experimental studies.Curr Drug Targets 2014, 15:551-557.

62. Sarkar C, Zhao Z, Aungst S, Sabirzhanov B, Faden Al, Lipinski MM: Impaired autophagy flux is associated with neuronal cell death after traumatic brain injury.Autophagy 2014, 10:2208-2222.

\section{Tables}

Table 1. qPCR primers and immuno-labeling reagents. 


\begin{tabular}{|c|c|c|c|}
\hline Antigen & Host, dilution & Vendor & Catalog number \\
\hline \multicolumn{4}{|l|}{ qPCR primers } \\
\hline Clec7a (isoform 1) & Mouse & Applied Biosystems & Mm01183349_m1 \\
\hline Clec7a (isoform 2) & Mouse & Applied Biosystems & Mm01183350_m1 \\
\hline Ncf1 & Mouse & Applied Biosystems & Mm00447921_m1 \\
\hline Ctss & Mouse & Applied Biosystems & Mm01255859_m1 \\
\hline C1qa & Mouse & Applied Biosystems & Mm00432142_m1 \\
\hline $\mathrm{C} 1 \mathrm{qb}$ & Mouse & Applied Biosystems & Mm00437836_m1 \\
\hline C1qc & Mouse & Applied Biosystems & Mm00776126_m1 \\
\hline C3 & Mouse & Applied Biosystems & Mm00437838_m1 \\
\hline Itgam (Cd11b) & Mouse & Applied Biosystems & Mm00434455_m1 \\
\hline Cxcl10 & Mouse & Applied Biosystems & Mm00445235_m1 \\
\hline Pmp22 & Mouse & Applied Biosystems & Mm01333393_m1 \\
\hline Serpin3a & Mouse & Applied Biosystems & Mm00776430_m1 \\
\hline Arc & Mouse & Applied Biosystems & Mm01204954_g1 \\
\hline Egr1 & Mouse & Applied Biosystems & Mm00656724_m1 \\
\hline $\mathrm{C} 4 \mathrm{a}$ & Mouse & Applied Biosystems & Mm01132415_g1 \\
\hline Gbp2 & Mouse & Applied Biosystems & Mm00494576_g1 Mm04209424_g1 \\
\hline Trem2 & Mouse & Applied Biosystems & Mm00658576_m1 \\
\hline Cd74 & Mouse & Applied Biosystems & Mm03047343_m1 \\
\hline Cd68 & Mouse & Applied Biosystems & Mm00440207_m1 \\
\hline Psmb8 & Mouse & Applied Biosystems & Mm00525305_m1 \\
\hline Tmem119 & Mouse & Applied Biosystems & Mm01187858_m1 \\
\hline Egfr & Mouse & Applied Biosystems & Mm00438980_m1 \\
\hline Flt1 & Mouse & Applied Biosystems & Mm99999915_g1 \\
\hline Gapdh & Mouse & Applied Biosystems & \\
\hline
\end{tabular}

\section{Flow cytometry antibodies}




\section{Surface antigens}

CD45-eF450

CD11b-APCeF780

Ly6C-AF700

MHCl-PECy7

Intracellular staining

Ki67-PECy7

PCNA-AF647

CD68-PerCPCy 5.5

NeuN-PE

Vglut1-APC

Lamp1-PerCPCy5.5

Lamp2-PE

Sqstm1/p62-AF647

ATG5-AF647

ATG7-AF700

Ubiquitin-AF647

H3-AF647

Acetylated (Ac) Lysine (Lys)-PECy7

H3-Ac-Lys9-AF488

H3-Ac-Lys18-AF488

H3-Ac-Lys27-AF647

H3-Ac-Lys36-AF647

Phospho(ser149)-H2A.X-PECy7

p16-APC

p21-AF488
Mouse,

1:100

Mouse,

$1: 100$

Mouse, 1:50 Biolegend

Mouse,

1:100

Biolegend
eBioscience

eBioscience

128024

114616
48-0451-82

47-0112-82
Mouse,

1:100

Mouse, 1:50

Mouse,

1:100

Mouse,

1:100

Mouse,

1:100

Mouse,

1:100

Mouse,

$1: 100$

Mouse, 1:20

Mouse, 1:50

Mouse, 1:50

Mouse,

$1: 100$

Mouse,

1:100

Mouse,

Mouse, 1:20

1:100
Biolegend

Biolegend

Biolegend

Millipore Sigma

StressMarq

Biolegend

Biolegend

Novus Biologicals

Biolegend

R\&D Systems

Biolegend

Cell Signaling

Technology

Biolegend

Cell Signaling

Technology

Cell Signaling

Technology

Cell Signaling

Technology
623408

652426

307912

137010

FCMAB317PE

SMC-394D-APC

121626

108506

NBP142822AF647

847410

FAB6608N

838710

$12230 \mathrm{~S}$

9683S

$73508 S$

$39030 S$

$84061 S$

613420 


\begin{tabular}{|c|c|c|c|}
\hline TNF-PECy7 & $\begin{array}{l}\text { Mouse, } \\
1: 100\end{array}$ & $\begin{array}{l}\text { Cell Signaling } \\
\text { Technology }\end{array}$ & \\
\hline IL-1ß-PerCPeF710 & Mouco & Binlegend & SPC-1280D-APC \\
\hline Brefeldin A & $1: 100$ & & $\begin{array}{l}\text { NBP2- } \\
\text { 43697AF488 }\end{array}$ \\
\hline & $\begin{array}{l}\text { Mouse, } \\
1: 100\end{array}$ & StressMarq & 506324 \\
\hline 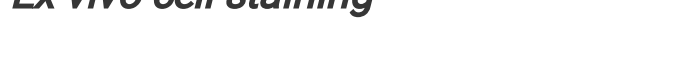 & Mouse, 1:20 & RND & $46-7114-82$ \\
\hline & & Biolegend & 420601 \\
\hline & Mouse, & eBioscience & \\
\hline & $\begin{array}{l}\text { Mouse, } \\
\text { 1:100 }\end{array}$ & Biolegend & \\
\hline & Mouse, 1:50 & & \\
\hline & Mouse, 1:50 & & \\
\hline & $\begin{array}{l}\text { Mouse, } \\
1: 1000\end{array}$ & & \\
\hline H2dcfda (DCF) & $8 \mu \mathrm{M}$ & ThermoFisher & D399 \\
\hline LysoTracker Deep Red & $75 \mathrm{nM}$ & ThermoFisher & L12492 \\
\hline Cyto-ID Autophagy Detection Kit & 1:1000 & Enzo Life Sciences & $\begin{array}{l}\text { ENZ-51031- } \\
\text { K200 }\end{array}$ \\
\hline ProteoStat Aggresome Detection Kit & & & \\
\hline BODIPY 493/503 & $1: 1000$ & Enzo Life Sciences & \\
\hline Lipi-Blue & & & K100 \\
\hline FluoroMyelin Red & $2 \mu \mathrm{M}$ & ThermoFisher & \\
\hline FerroOrange & $0.5 \mu \mathrm{mol} / \mathrm{L}$ & Dojindo & D3922 \\
\hline MitoSpy Red CMXRos & $1: 300$ & ThermoFisher & LD01-10 \\
\hline Glucose Uptake Assay Kit & $1 \mu \mathrm{mol} / \mathrm{L}$ & Dojindo & F34652 \\
\hline Biotracker ATP-Red & $25 \mathrm{nM}$ & Biolegend & F374-12 \\
\hline BODIPY-Pepstatin A & $200 \mu \mathrm{g} / \mathrm{mL}$ & $\begin{array}{l}\text { Cayman Chemical } \\
\text { Company }\end{array}$ & 424802 \\
\hline $\begin{array}{l}\text { LysoLive }{ }^{T M} \text { Lysosomal Acid Lipase } \\
\text { Assay Kit }\end{array}$ & & Millipore Sigma & 600470 \\
\hline & $1 \mu \mathrm{M}$ & $\begin{array}{l}\text { ThermoFisher } \\
\text { Abcam }\end{array}$ & SCT045 \\
\hline
\end{tabular}




\begin{tabular}{|llll|}
\hline NeuN & Mouse, 1:500 & Millipore & MAB377 \\
LC3 & Rabbit, 1:200 & Novus Biologicals & NB100-2220 \\
P62 & Guinea Pig, 1:500 & Progen & GP62-C \\
Iba-1 & Rabbit, 1:200 & Cell Signaling Technology & 17198S \\
& - & - & \\
\hline
\end{tabular}

Figures 


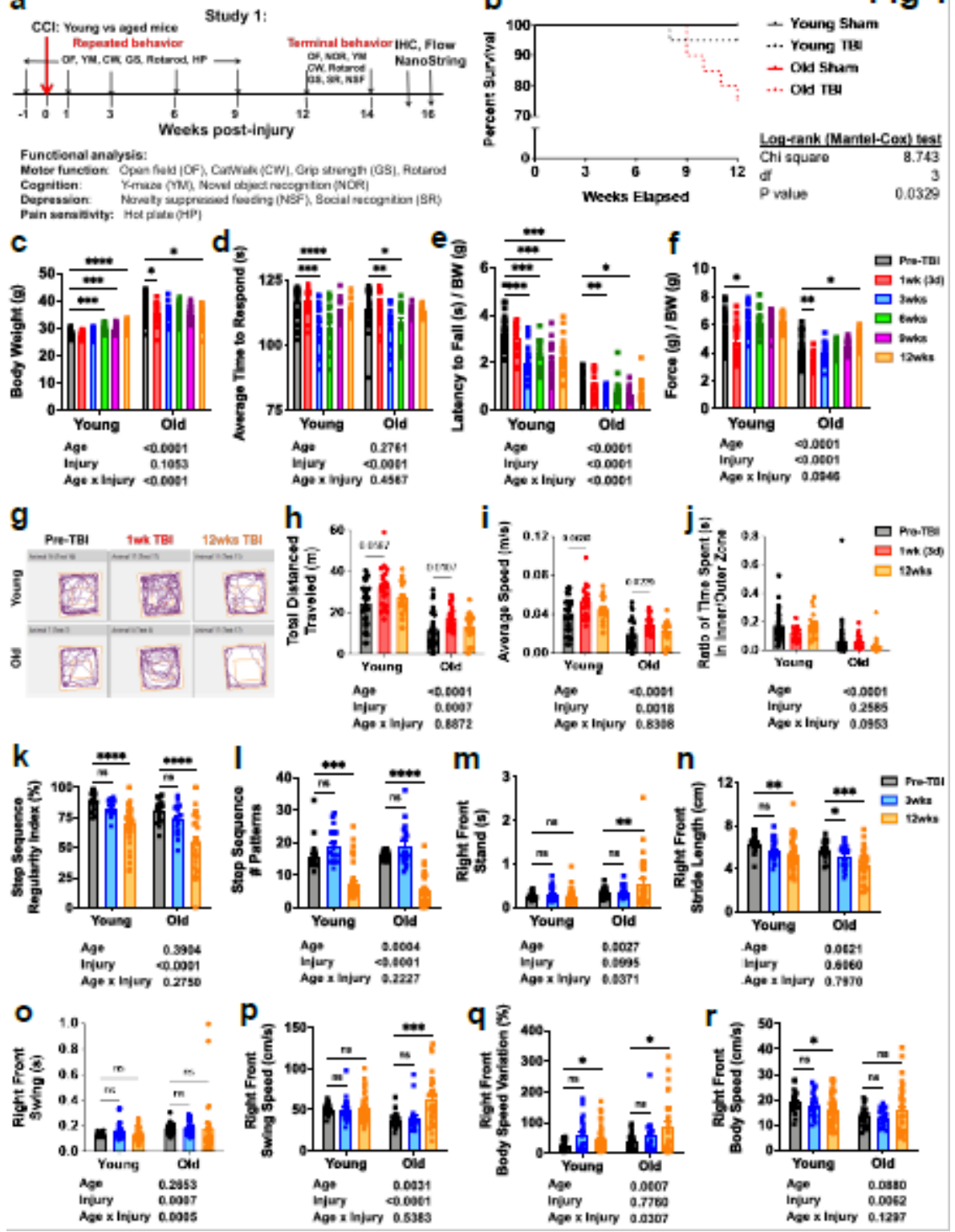

Figure 1

Effects of age on long-term survival and motor recovery after TBI. (a) Schematic diagram illustrating timepoints by weeks pre- or post-injury and of repeated or terminal behavior experiments conducted throughout study 1. Baseline behavior testing aimed at assessment of motor, cognition, and pain sensitivity was performed at $1 \mathrm{w}$ before injury, followed by a moderate controlled cortical impact (CCI) injury on the left cerebral cortex of mice randomly designated to the injury groups. Repeated behavior tests were performed on 1, 3, 6 and 9w post-injury. Terminal behavior for motor, cognitive, and depressionlike behavior were carried out between 12 and $14 \mathrm{w}$ post-injury before euthanizing the mice for immunohistochemistry, flow cytometry, and NanoString Analysis. (b) Survival plot of young and old mice in weeks elapsed for study 1. Compared to Old Sham $(n=12)$ and Young Sham $(n=13)$, both TBI injury groups ( $n=20$ /group) show decreased survival rates, with Old TBI group having the lowest survival rate of all mice. Kaplan-Meier survival curves were analyzed using the log-rank Mantel-Cox test. (c) Graph depicting body weight data of young and old mice at baseline and follow-up measurements post-injury. 
(d) Graph depicting latency time to withdrawal in hot plate test for thermal sensitization. Both age groups displayed thermal hyperalgesia between 3-6w post-injury, but no age effect was observed. (e) Graph depicting rotarod data from baseline to $12 \mathrm{w}$ post-injury. (f) Graph depicting grip strength measurements from baseline to $12 \mathrm{w}$ post-injury. $(\mathrm{g})$ Representative graph of spontaneous movement by young and old mice in the open field test at baseline, $3 w$ and $12 w$ after TBI. (h-j) Spontaneous locomotor activity in an open field apparatus was recorded and analyzed with use of the AnyMaze animal behavior system. Injury effects could be observed in two parameters: total distance travelled (h) and average speed (i). Age effects could be observed in all three parameters depicted. (k-r) Graphs depicting parameters of CatWalk gait analysis tested at baseline, 3 and 12w post-injury. 2-way ANOVA followed by Tukey's post-hoc test. $\star \star \star * * p<0.0001, * \star * p<0.001, * * p<0.01, * p<0.05$.
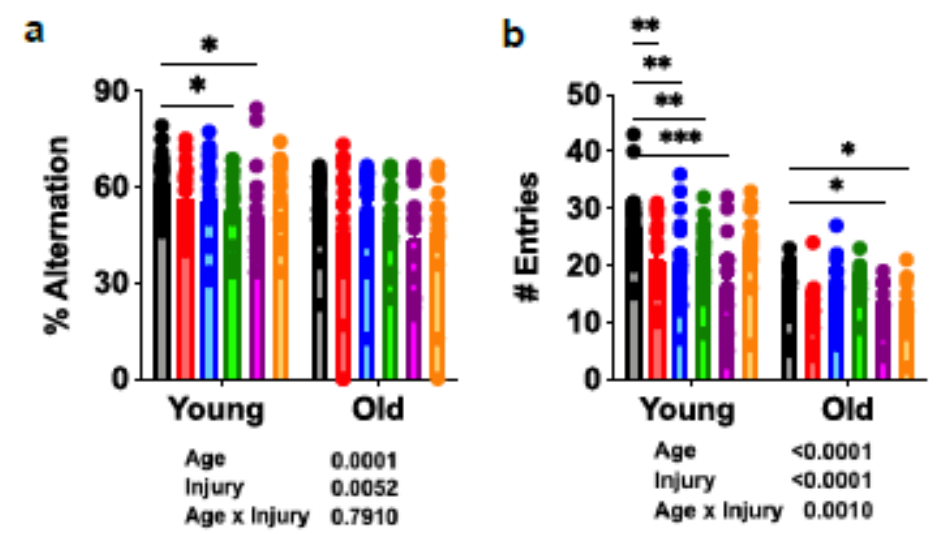

Fig 2

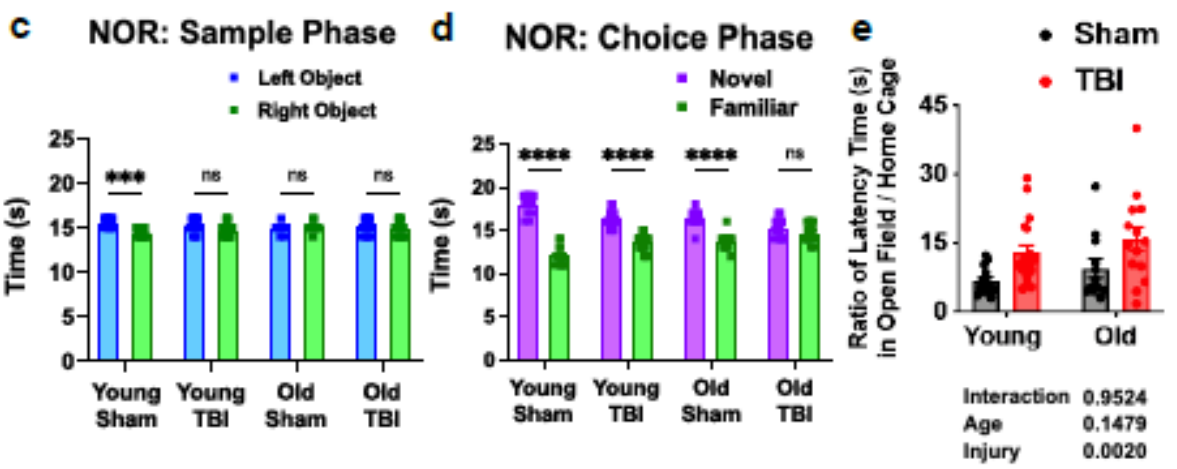

f SR: Sequence 1-Index

g SR: Sequence 2-Index

h SR: Sequence 3-Index
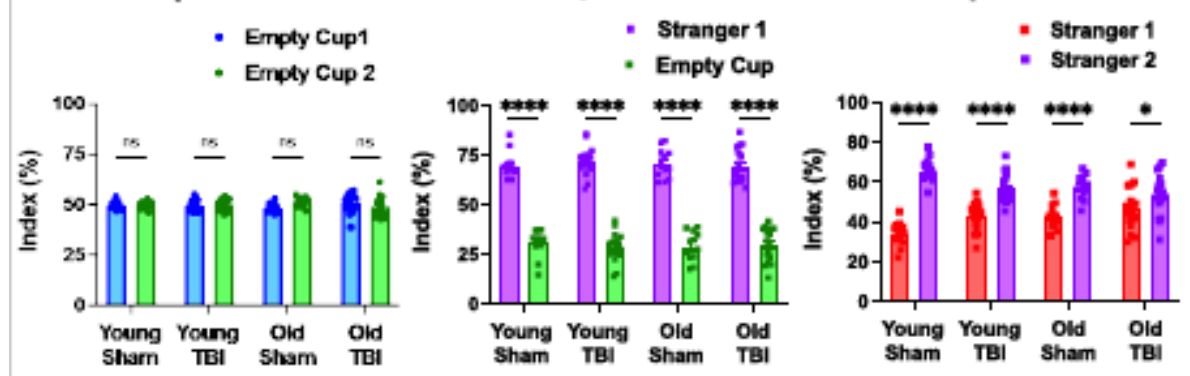

Figure 2

Effects of age on long-term cognition, depression, and social behavior. (a-b) Graph depicting percentage of alternation and total arm entry numbers for young and aged mice in baseline and repeated behavior tests. Significant effects of age and injury could be observed in both parameters observed. (c) During the 
sample phase of the NOR task, no differences between groups were seen in exploration time between left and right-side objects. (d) Graph depicting time spent exploring the novel versus familiar object during the choice phase of the NOR experiment. All groups except Old TBI showed significant preference for novel object over familiar object. (e) Ratio of latency time to food pellet in open field versus home cage showed injury effects for both age groups, but no aged effects were observed. (f-h) Graphs depicting preference index of each sequence for the social recognition experiment are shown. $\mathrm{N}=13$ (Young Sham), 12 (Old Sham), 19 (Young TBI), and 17 (Old TBI). 2-way ANOVA followed by Tukey's post-hoc test. ${ }^{\star \star \star \star} \mathrm{p}<0.0001$, *** $p<0.001, * * p<0.01, * p<0.05$.
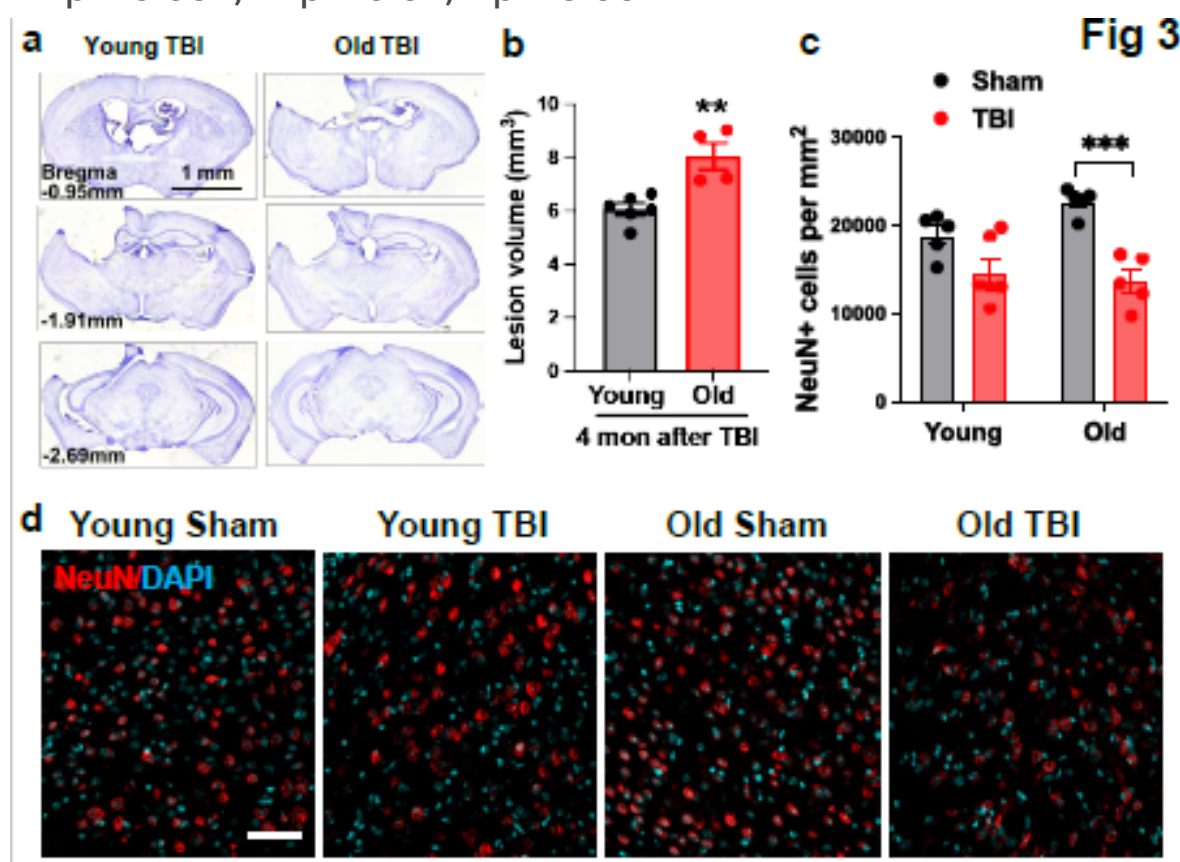

Old Sham

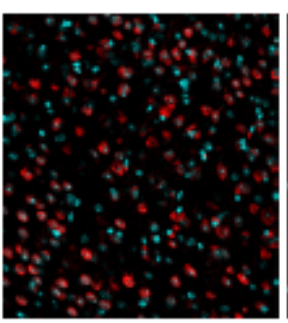

Old TBI
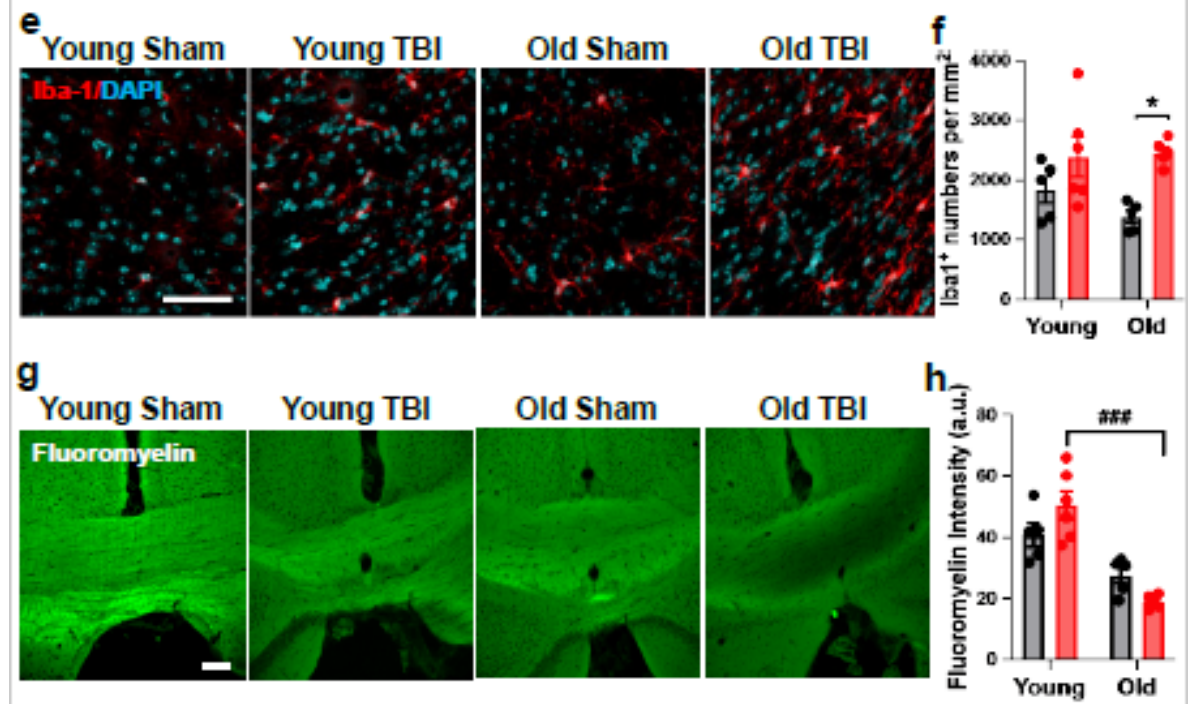

\section{Figure 3}

Aged mice exhibit exacerbated tissue damage, higher neuronal loss and exaggerated white matter degradation after injury. (a) Representative images from lesion site of Old TBI and Young TBI mice at 16w after injury. (b) Lesion volume was quantified based on the Cavalieri method of unbiased stereology using Stereologer 2000 software. $N=6$ (Young TBI) and 4 (Old TBI). Unpaired t test, $p<0.01$. (c) Neuronal 
count of NeuN-positive cells within the ipsilateral cortex showed significant neuronal loss in Old TBI mice compared to Old Sham group. (d) Representative images of NeuN and DAPI. (e-f) Representative images and quantified lba-1-positive cells. (g-h) Representative images and quantification data for Fluoromyelin staining in the medial corpus collosum. $\mathrm{N}=5$ (Young Sham), 5 (Old Sham), 6 (Young TBI), and 5 (Old TBI). 2-way ANOVA followed by Tukey's post-hoc test. ${ }^{* \star *} p<0.001,{ }^{*} p<0.05$, vs Old Sham; \#\#\# $p<0.001$, vs Young TBI.
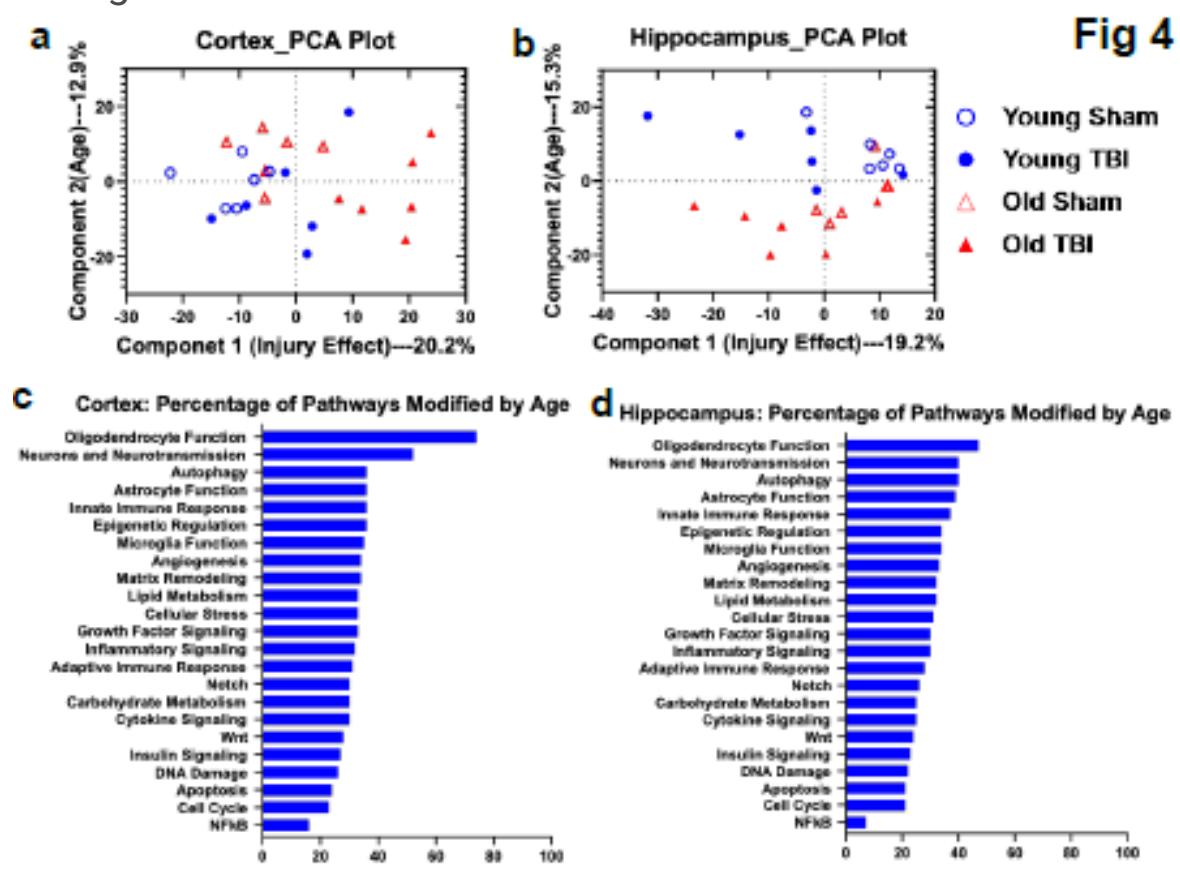

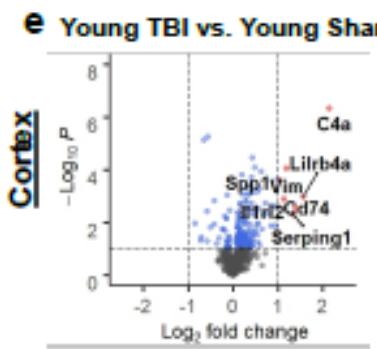

h Young TBI vs. Young Sham

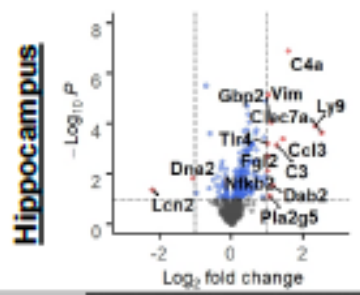

f Old TBI vs. Old Sham

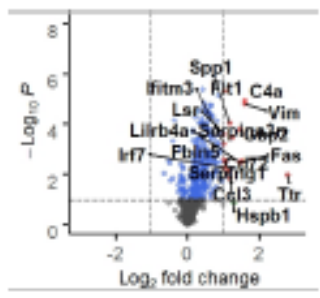

i Old TBI vs. Old Sham

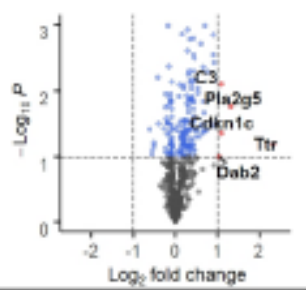

g Old TBI vs. Young TBI

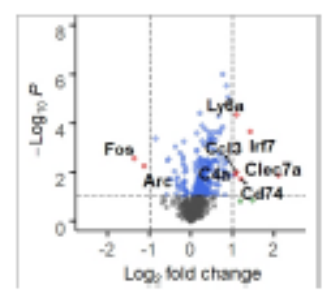

j Old TBI vs. Young TBI

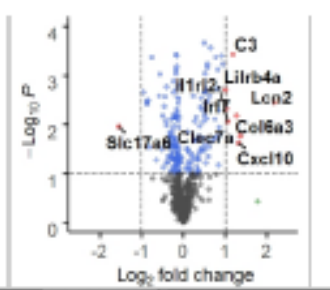

Figure 4

NanoString analysis of ipsilateral cerebral cortex and hippocampus at $16 \mathrm{w}$ following TBI. (a) PCA plot of the transcription levels of neuroinflammatory genes in the cortex. Principal component analysis (PCA) was performed using all normalized gene counts from the NanoString Neuroinflammation panel. The four sample groups were Young Sham (open circle), Young TBI (closed circle), Old Sham (open triangle) and Old TBI (closed triangle). Injury-related effects were captured on component 1, separating the TBI groups from Sham groups from left to right along the x-axis. Age-related effects were captured on 
component 2, separating the young adult groups from aged groups. (b) PCA plot of ipsilateral hippocampus tissue samples. (c-d) Pathway analysis of transcriptome levels in the cortex and hippocampus based on gene annotations given by NanoString reveal high percentage of genes related to oligodendrocyte function being modified by age. (e-g) Volcano plot of differentially expressed genes in the cortex, demonstrating specific genes with log2 (fold change) larger than 1 and statistical significance of $\log 10(\mathrm{P})$ higher than 1. ( $\mathrm{h}-\mathrm{j})$ Volcano plot of differentially expressed genes in the hippocampus, demonstrating specific genes with log2 (fold change) larger than 1 and statistical significance of $\log 10(P)$ higher than 1. $n=6$ mice/group.

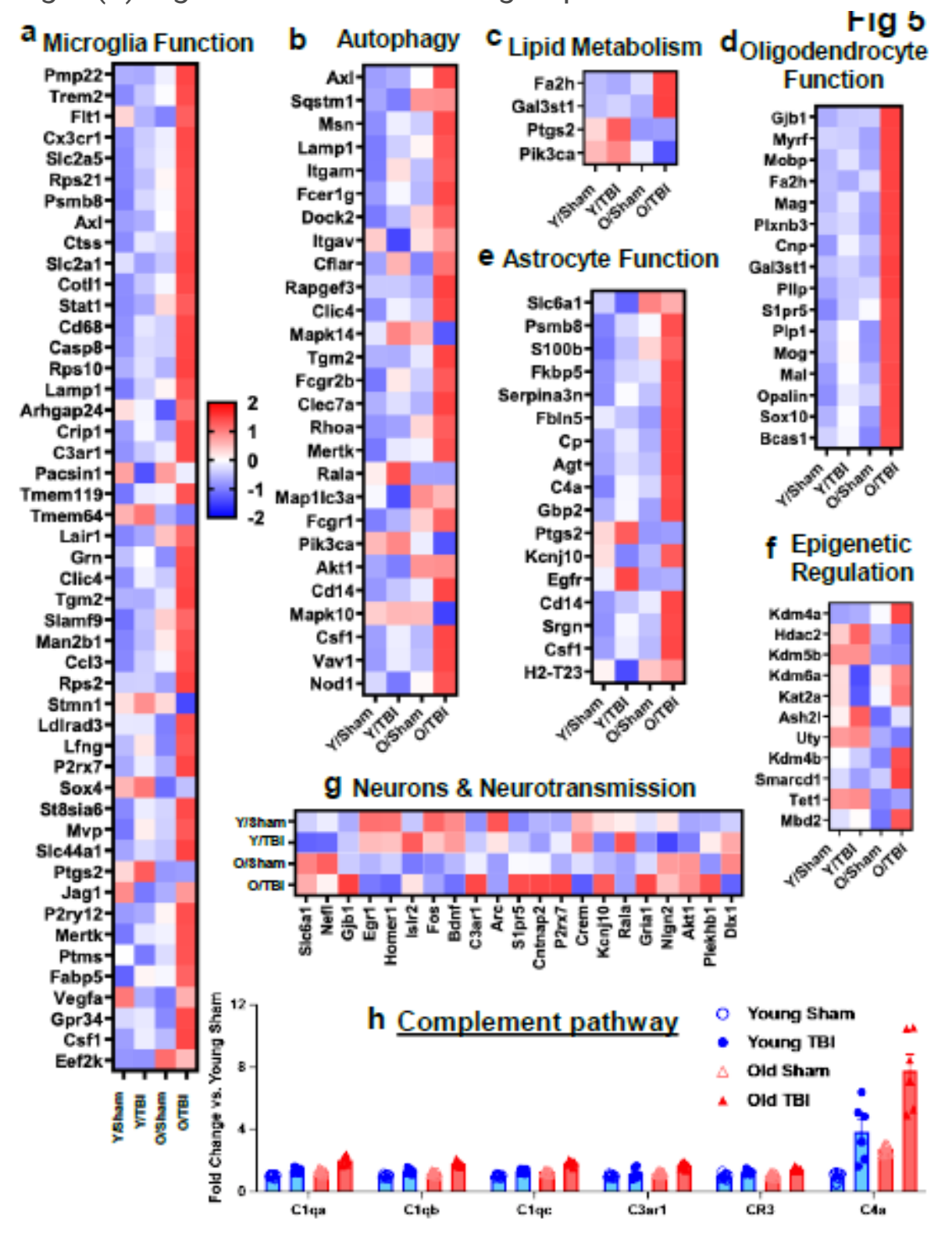

Figure 5

Age significantly alters neuroinflammatory profile of the injured cerebral cortex at the transcriptome level. (a-g) Heatmap of genes related to microglia function (a), autophagy (b), lipid metabolism (c), oligodendrocyte function (d), astrocyte function (e), epigenetic regulation (f), and neurons and neurotransmission $(\mathrm{g})$ that were altered in differential expression analysis between Old TBI and Young 
TBI, i.e., genes only altered in Set 4. Color coding was based on z-score scaling. (h) Bar graph of genes within the complement pathway that showed differential expression. $n=6$ mice/group.

Fig 6

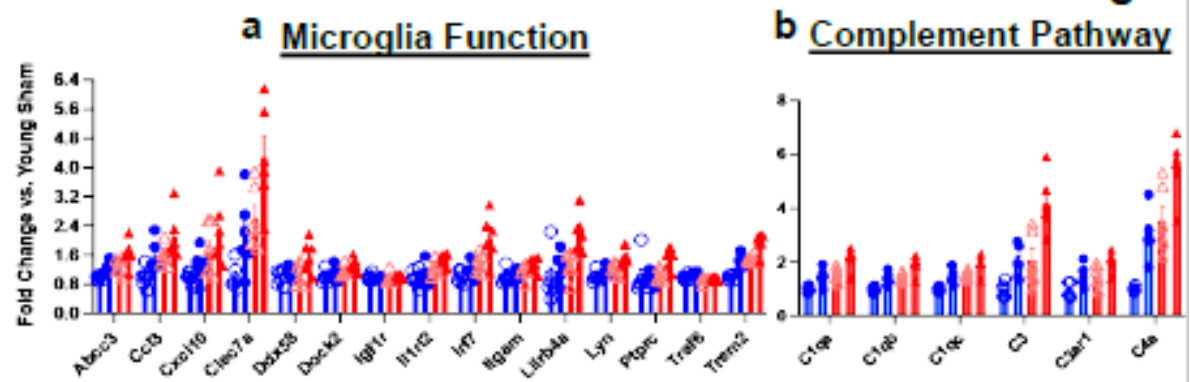

c Autophagy Regulation

d Epigenetic Regulation

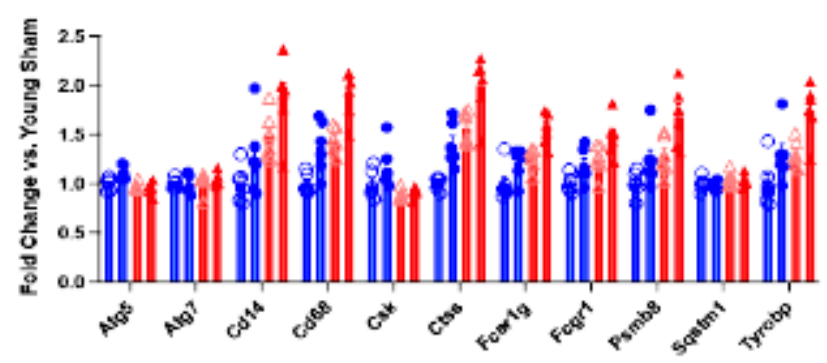

${ }^{e}$ Astrocyte Function $f$ Oligodendrocyte Function
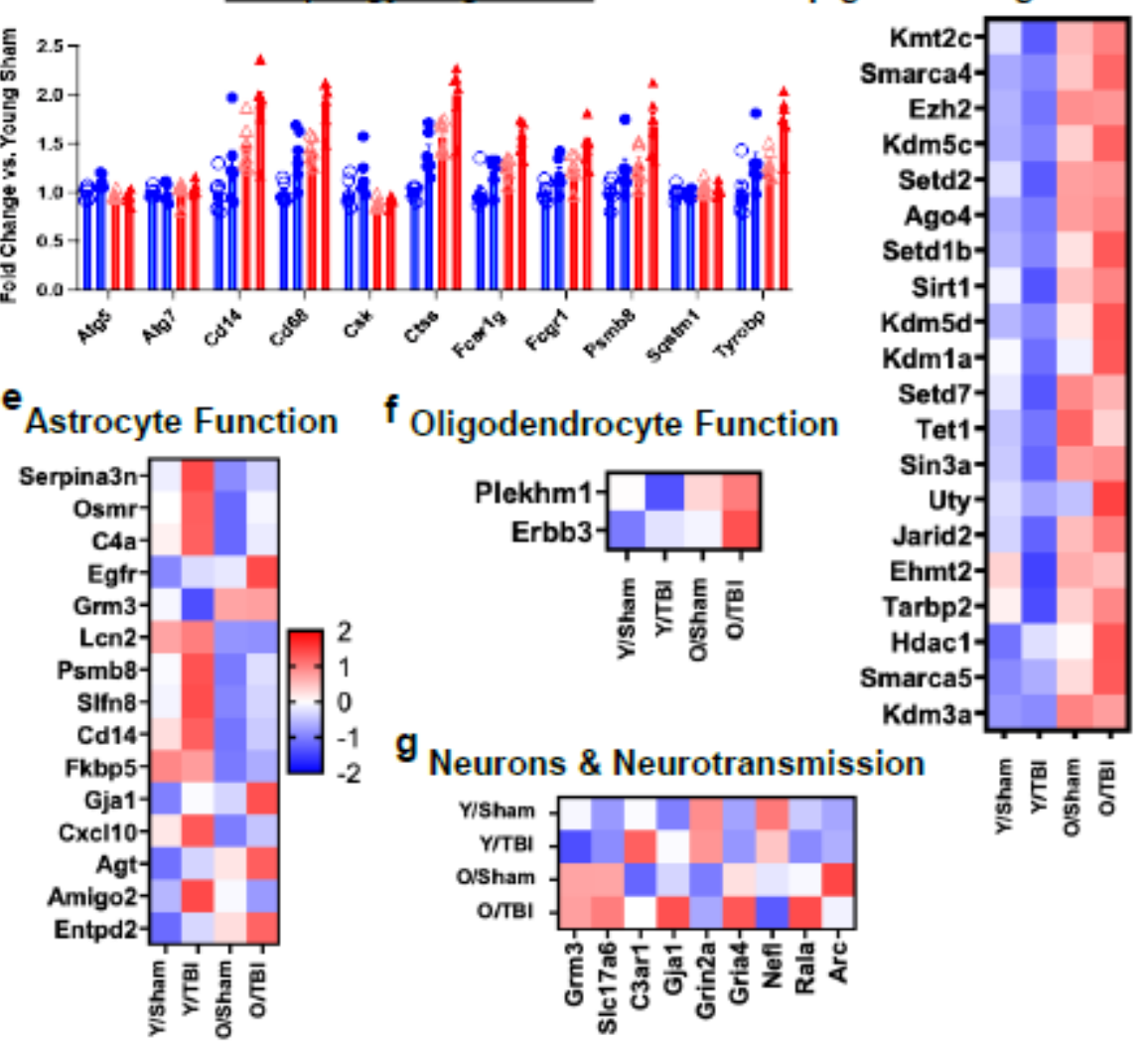

geurons \& Neurotransmission
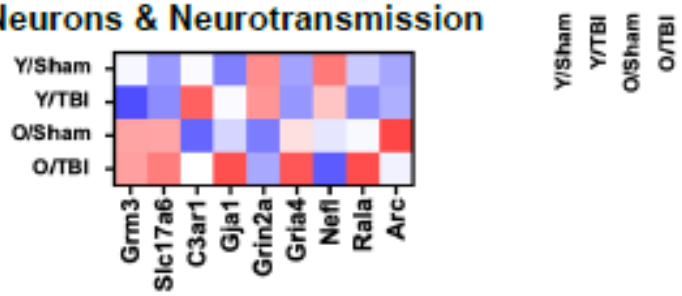

Figure 6

Age-related transcriptomic changes in neuroinflammatory genes of the hippocampus at $16 \mathrm{w}$ post-injury. (a-c) Bar graph of genes related to microglia function (a), the complement pathway (b), and autophagy (c) that showed differential expression in pairwise comparisons between Old TBI and Young TBI. (d-g) Heatmap of differentially expressed genes related to epigenetic regulation (d), astrocyte function (e), oligodendrocyte function (f), and neurons and neurotransmission (g) when comparing between Old TBI and Young TBI, i.e., genes only altered in Set 4. Color coding was based on z-score scaling. $n=6$ mice/group. 
TBI

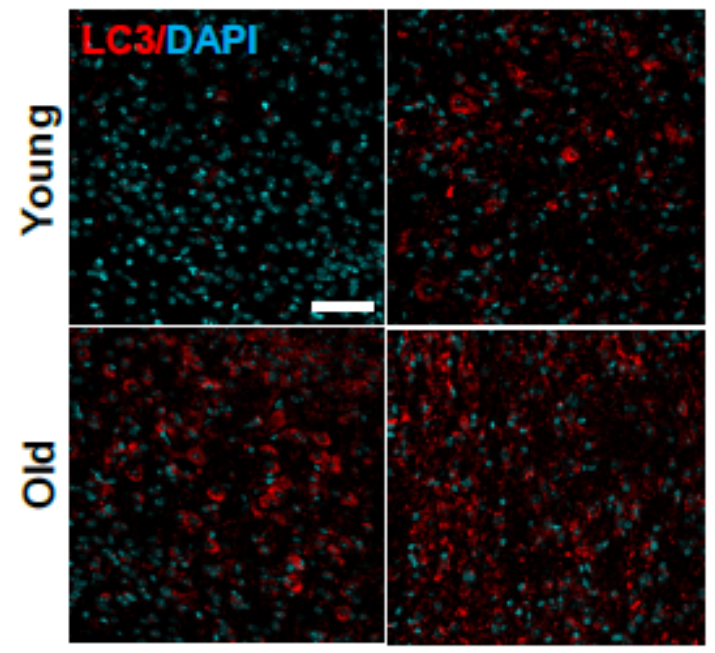

b - Sham

- TBI

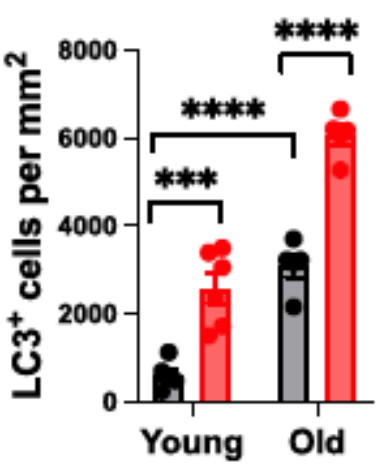

C

TBI
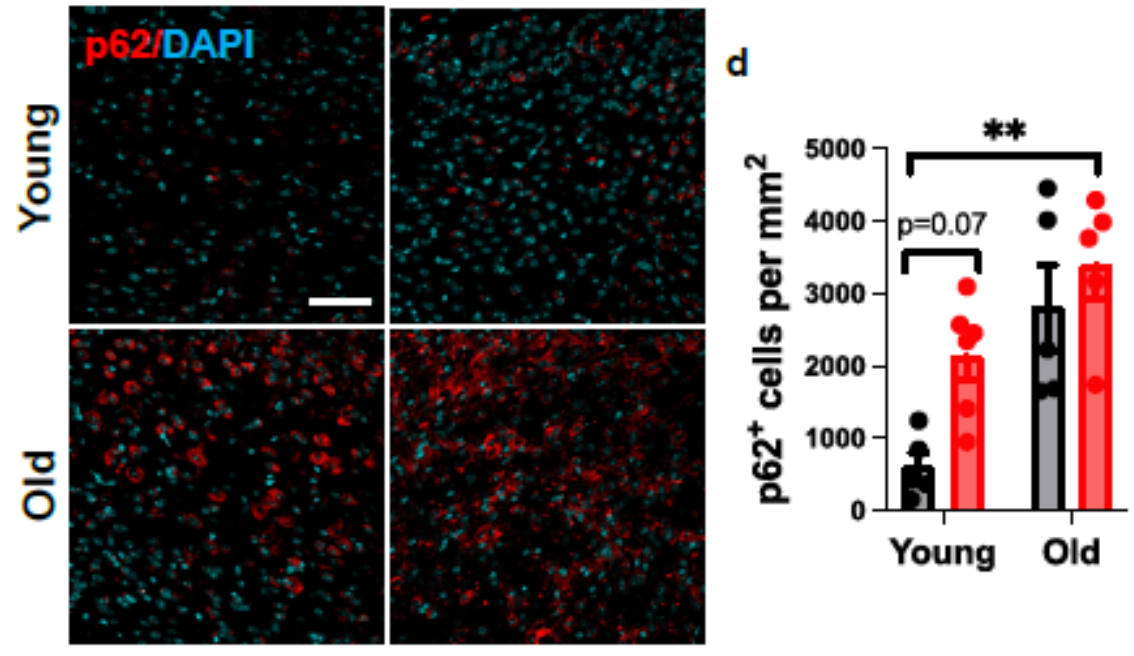

Figure 7

The aged TBI brain displays increased expression of autophagy markers during the chronic phase of injury. (a-b) Representative images and quantified data of LC3-positive cells in the ipsilateral cerebral cortex at $16 \mathrm{w}$ post-injury. (c-d) Representative images and quantification of p62/SQSTM1-positive cells in the ipsilateral cerebral cortex at 16 w post-injury. $\mathrm{N}=5$ (Young Sham), 5 (Old Sham), 6 (Young TBI), and 5 (Old TBI). 2-way ANOVA followed by Tukey's post-hoc test. ${ }^{\star \star \star *} p<0.0001,{ }^{\star \star *} p<0.001,{ }^{\star \star} p<0.01$. 

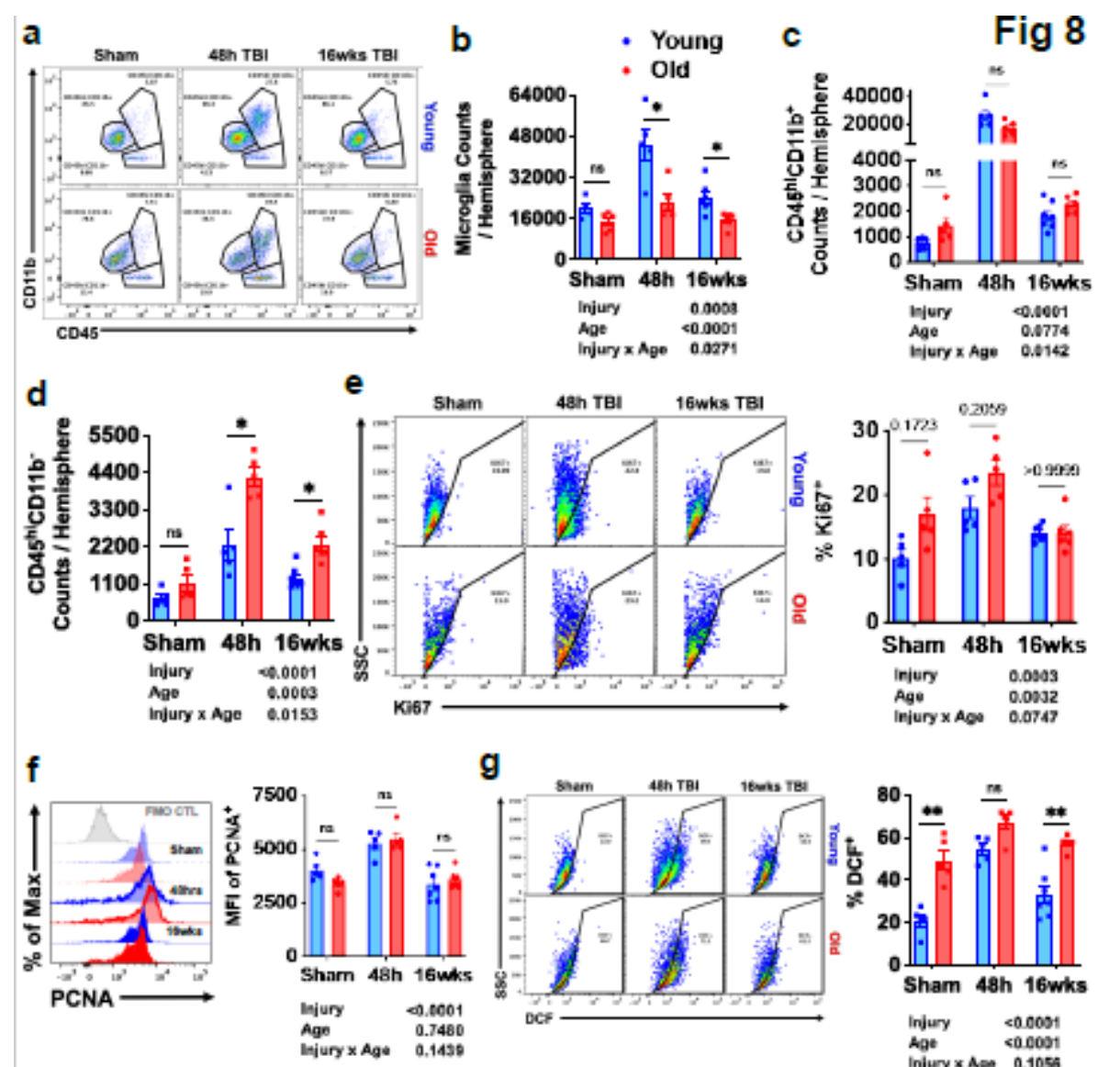

g
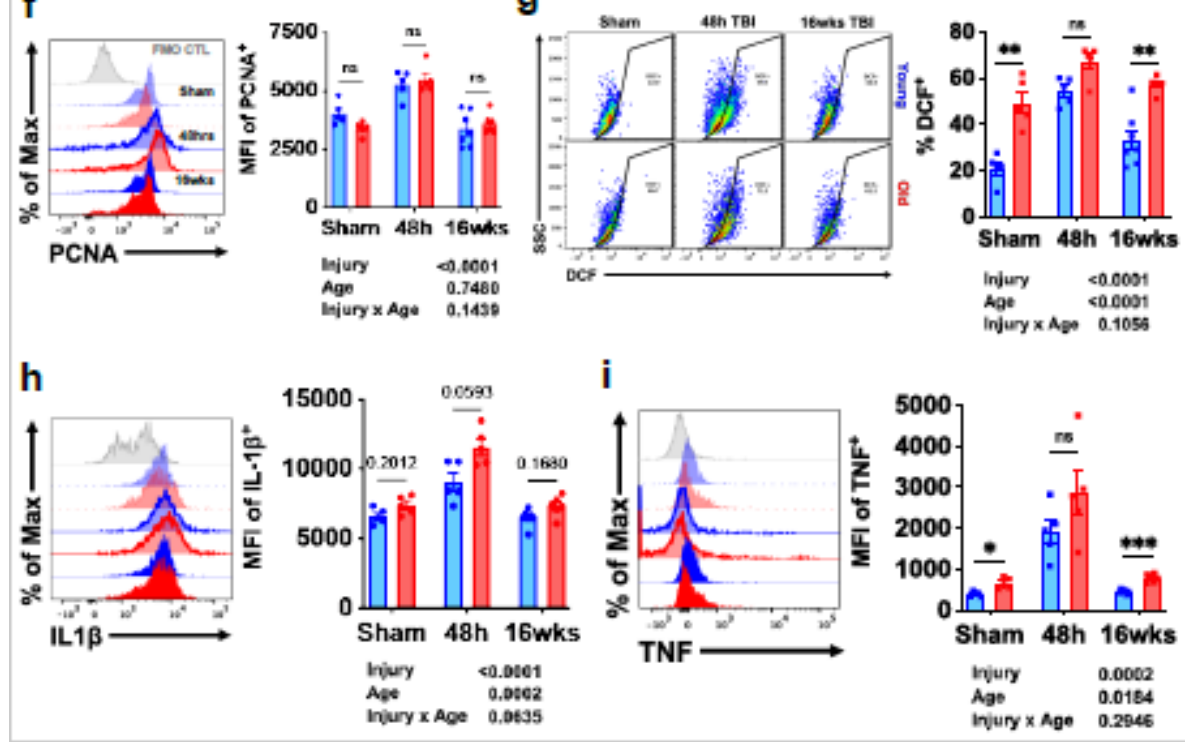

Figure 8

The effects of old age on the central immune response to TBI. (a) A representative dot plot of leukocyte populations in the brain at $48 \mathrm{~h}$ and $16 \mathrm{w}$ after TBI. Quantification of CD45intCD11b+ microglia (b), CD45hiCD11b+ myeloid cells (c), and CD45hiCD11b- putative lymphocyte (d) cell counts are shown. Microglial proliferation as measured using cell cycle markers. A group effect of injury was seen in Ki67 (e) and PCNA (f) expression, driven largely by the increase at $48 \mathrm{~h}$ after TBI. Reactive oxygen species (ROS) and oxidative stress were measured using DCF. Group effects of age and injury were seen in microglial ROS levels (g). Pro-inflammatory cytokine production was assessed. The relative expression level of IL-1 $\beta$ (h) and TNF (i) in microglia is shown. For all histograms, gray = FMO control, blue = young, red $=$ old, Sham $=$ no outline/no fill, $48 \mathrm{~h} \mathrm{TBI}=$ bold outline/no fill, and $16 \mathrm{w} \mathrm{TBI}=$ bold outline/bold fill . $\mathrm{N}=5-7 /$ group. Data were analyzed using 2-way ANOVA group analysis with Tukey's test for multiple comparisons. ${ }^{\star \star \star} p<0.001,{ }^{\star \star} \mathrm{p}<0.01,{ }^{*} \mathrm{p}<0.05$. 

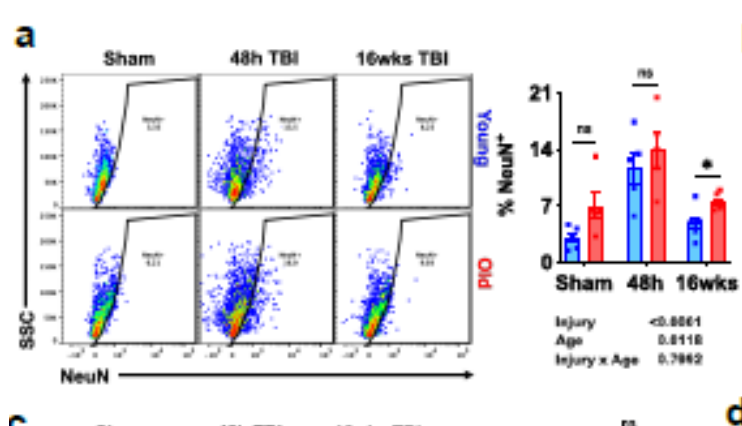

b

Fig 9

$c$
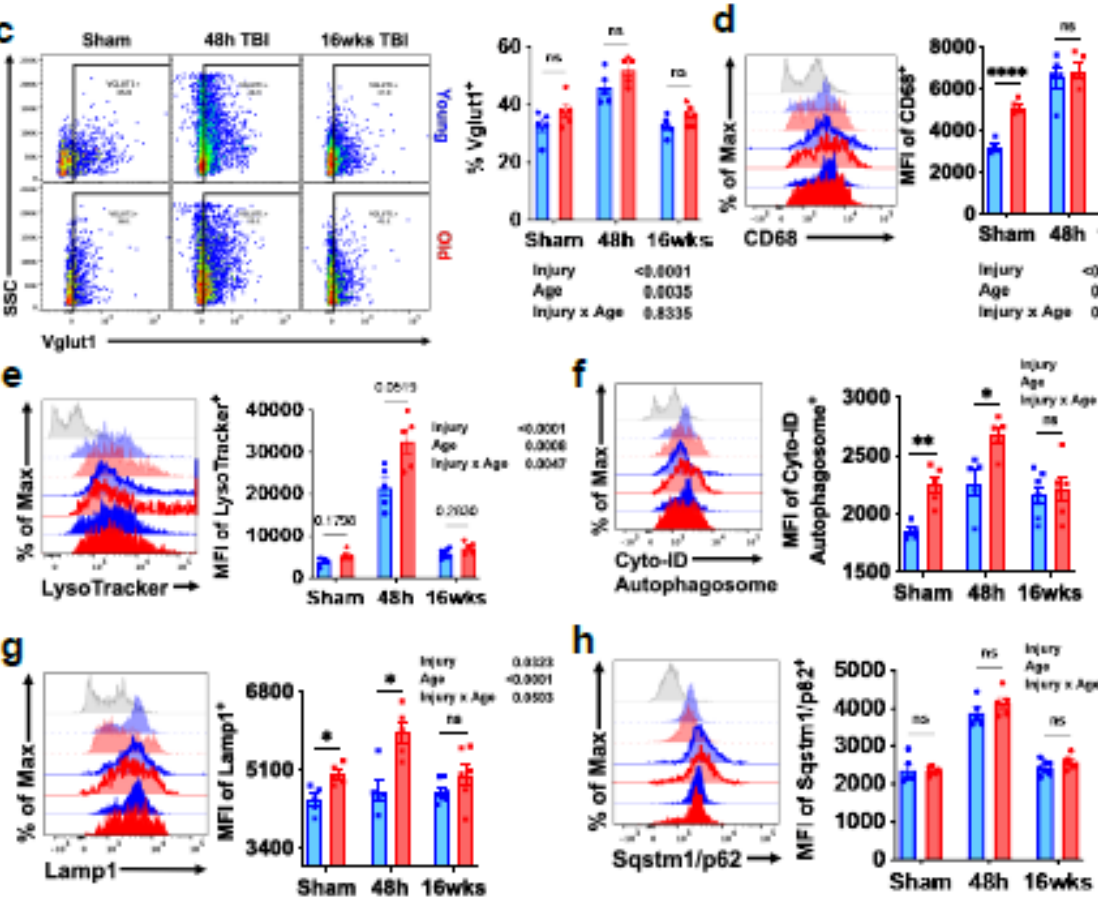

h
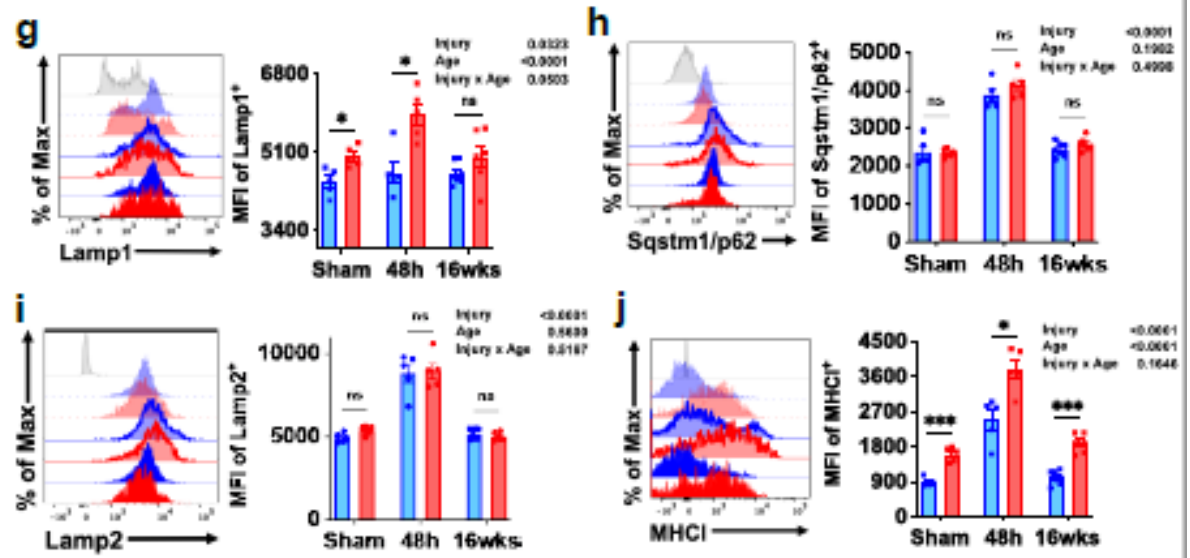

is men consens
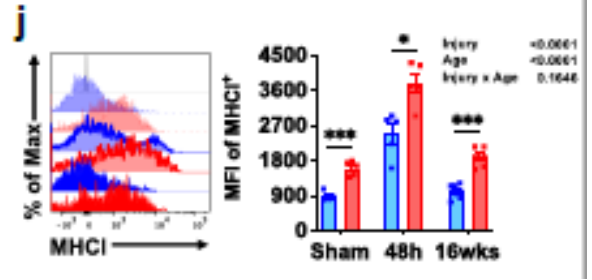

Figure 9

Microglial phagocytosis of neurons, expression of autophagy markers, and lipofuscin content is chronically increased with age and injury. Phagocytosis was assessed by intracellular detection of neuronal and myelin antigens. (a) The percentage of NeuN-positive microglia was significantly increased with age and injury. The mean fluorescence intensity of Fluoromyelin Red staining was acutely increased in microglia from both age groups (b). The presence of vesicular glutamate transporter 1 (vGlut1) (c) and the phagosome marker CD68 (d) were increased in microglia with both old age and TBI. Representative histograms illustrate the relative abundance of lysosomes and LC3II-positive autophagosomes in microglia as measured by LysoTracker (e) and Cyto-ID Autophagosome dyes (f). For all histograms, gray $=\mathrm{FMO}$ control, blue $=$ young, red $=$ old, Sham $=$ no outline $/$ no fill, $48 \mathrm{~h} \mathrm{TBI}=$ bold outline $/$ no fill, and $12 \mathrm{w}$ $\mathrm{TBI}$ = bold outline/bold fill. The mean fluorescence intensity of Lamp1 (g), Sqstm1/p62 (h), Lamp2 (i), and $\mathrm{MHCl}(\mathrm{j})$ for microglia are shown. $\mathrm{N}=5-7 /$ group. Data were analyzed using 2-way ANOVA group analysis with Tukey's test for multiple comparisons. ${ }^{* \star *} p<0.0001,{ }^{*} p<0.01,{ }^{\star} p<0.05$. 


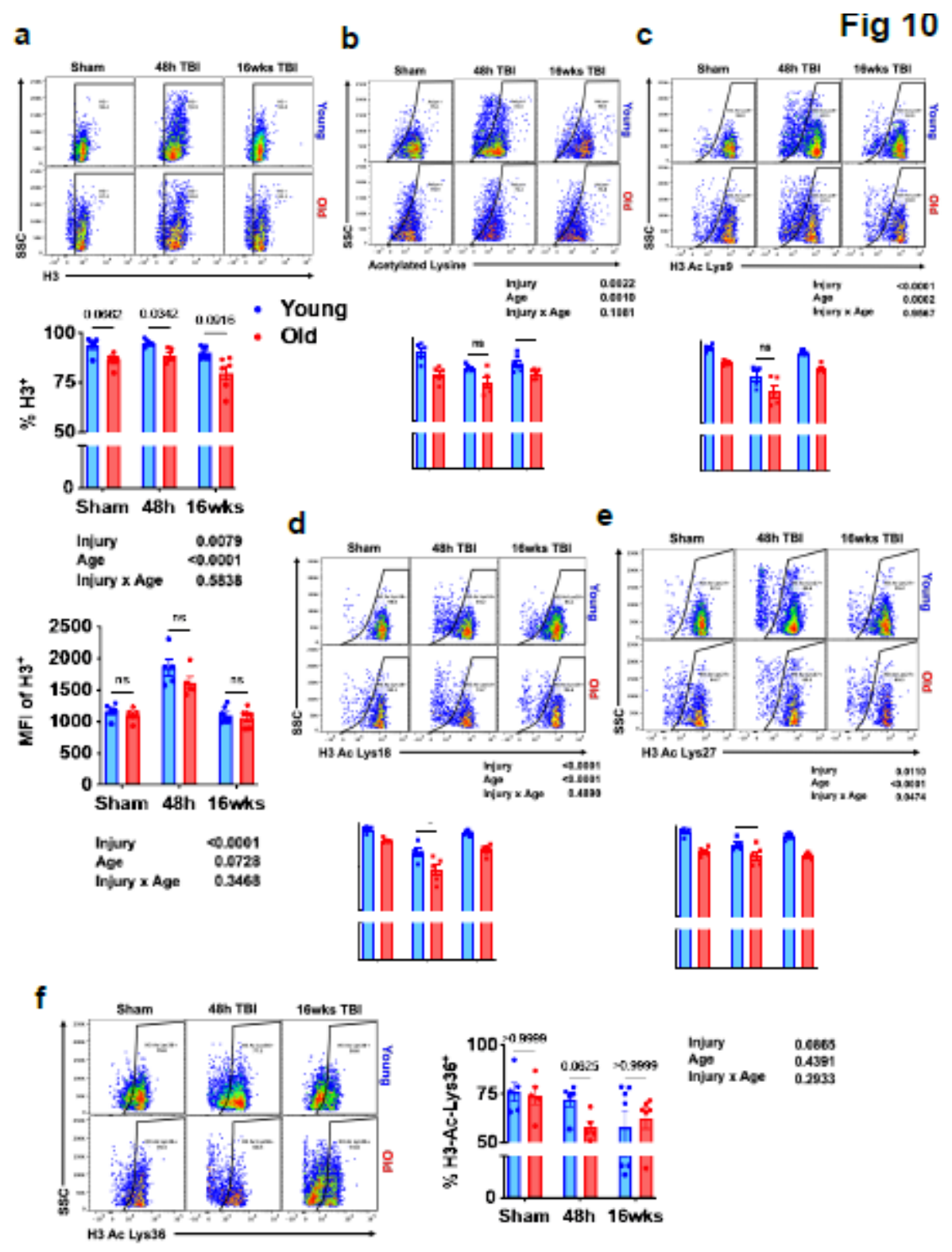

Figure 10

TBI exacerbates age-related alterations in histone acetylation of lysine residues in microglia. Flow cytometric analysis of epigenetic changes in microglia was performed. (a) The percentage of Histone 3 $(\mathrm{H} 3)$-positive microglia is shown in Sham control and at 48h and 16 w after TBI. The mean fluorescence intensity of $\mathrm{H} 3$ protein expression in microglia is also quantified. (b) Global lysine acetylation in microglia is illustrated in the representative dot plot. Acetylation frequencies of the H3 residues (c) Lysine 9, (d) Lysine 18, (e) Lysine 27, and (f) Lysine 36 were quantified. N=5-7/group. Data were analyzed using 2-way ANOVA group analysis with Tukey's test for multiple comparisons. ${ }^{\star \star \star *} p<0.0001,{ }^{* \star} p<0.001,{ }^{*} p<0.05$. 

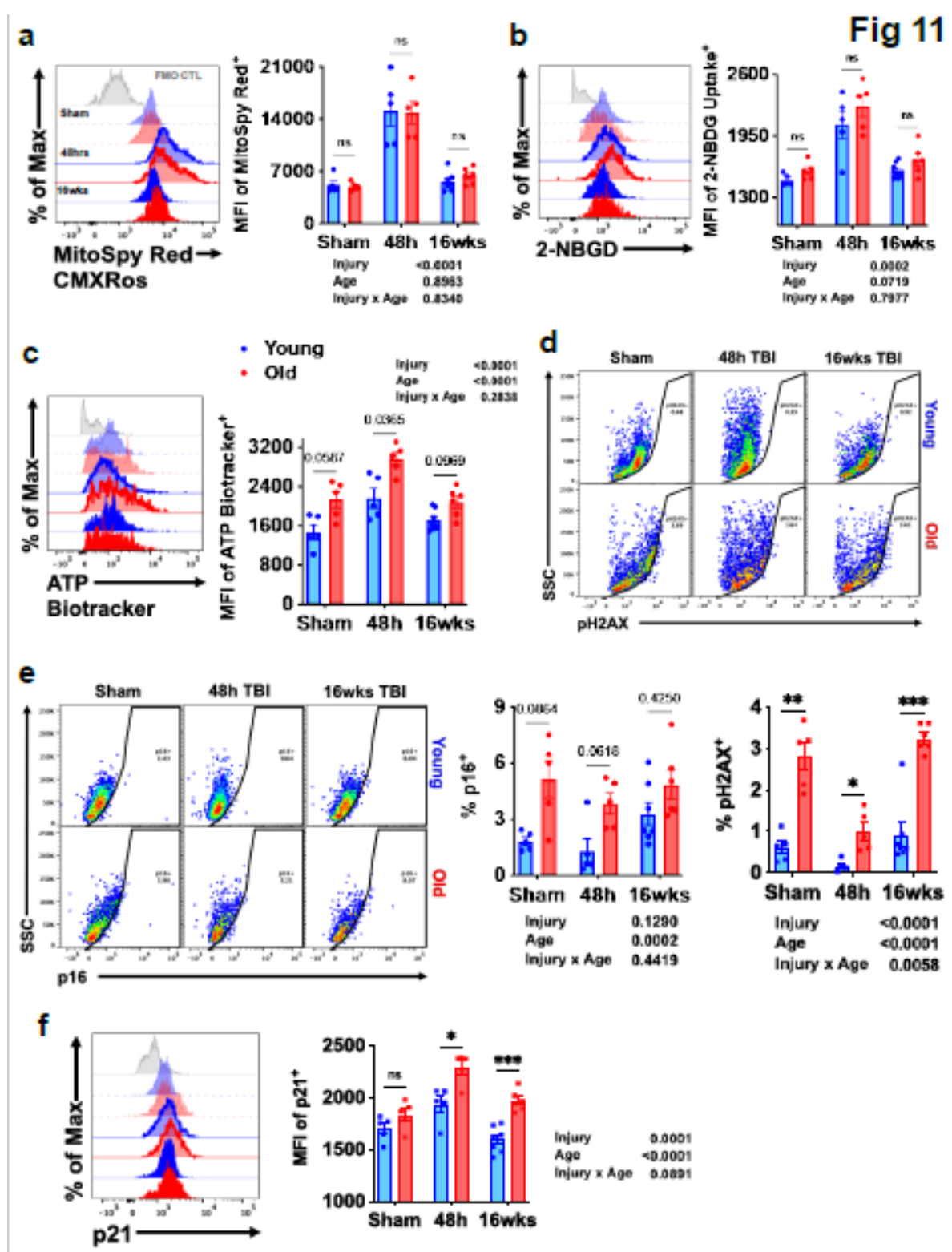

\section{Figure 11}

TBI exacerbates age-related alterations in metabolic activity and expression of senescence markers. Flow cytometric analysis of metabolic changes in microglia was performed. (a) Representative histogram depicts the relative mitochondrial membrane potential in microglia as measured using MitoSpy Red CMXRos. The mean fluorescence intensity of (b) Glucose uptake and (c) ATP levels in microglia were assessed using 2-NBDG and ATP Biotracker, respectively. (d) A representative dot plot illustrating the percentage of microglia immunoreactive to the DNA damage sensor, phosphorylated (ser139) H2AX, is shown and quantified. The frequency of (e) p16-positive and (f) p21-positive microglia was quantified. For all histograms, gray $=\mathrm{FMO}$ control, blue $=$ young, red $=$ old, Sham $=$ no outline $/$ no fill, $48 \mathrm{~h} \mathrm{TBI}=$ bold outline/no fill, and 16w TBI = bold outline/bold fill. N=5-7/group. Data were analyzed using 2-way ANOVA group analysis with Tukey's test for multiple comparisons. ${ }^{* \star *} p<0.0001,{ }^{* \star} p<0.001,{ }^{*} p<0.05$. 

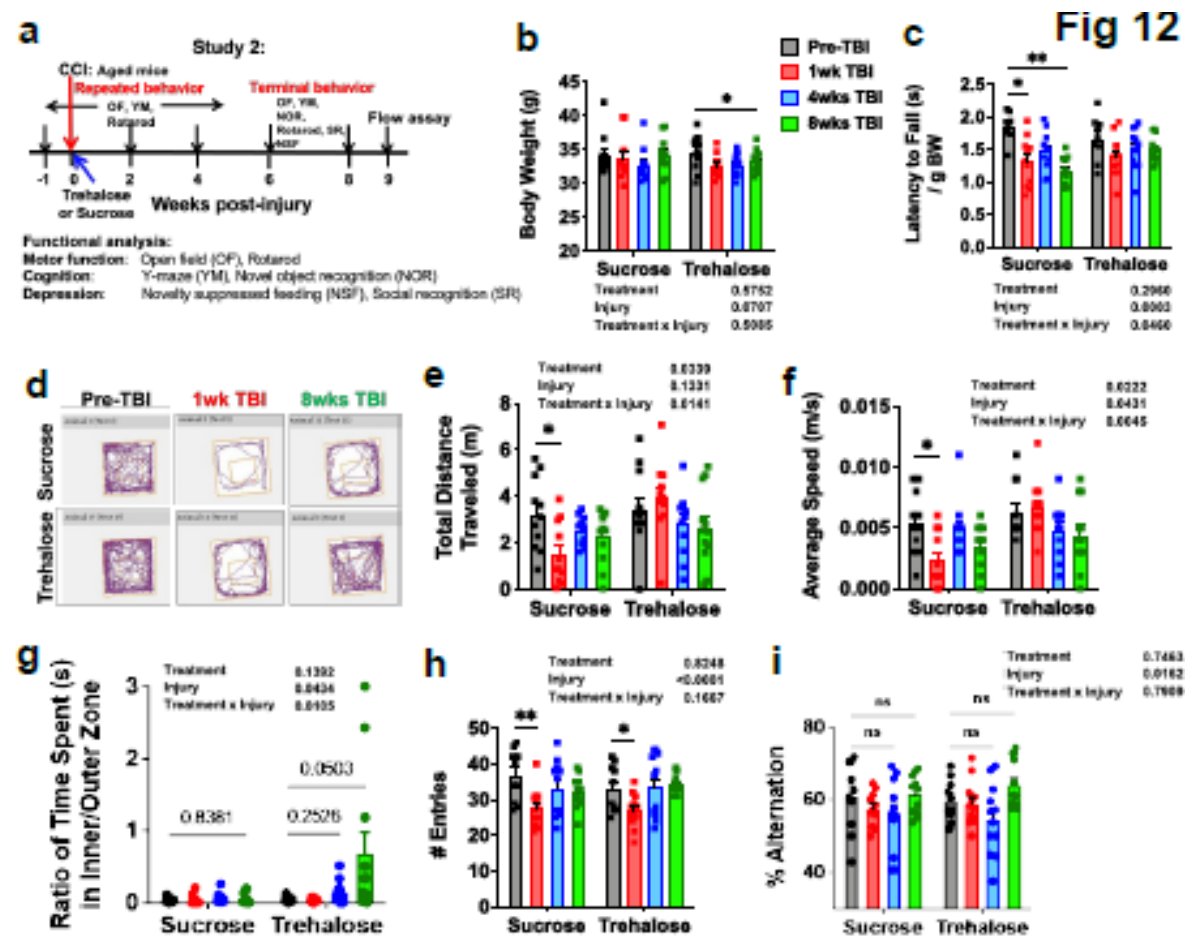

j NOR: Sample Phase
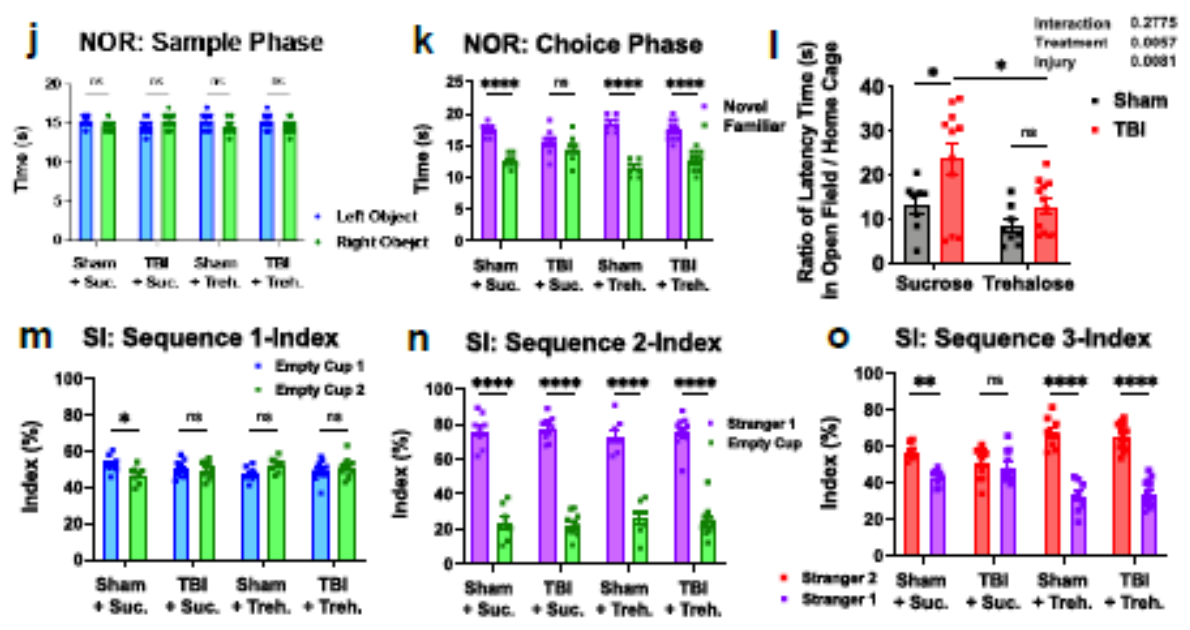

Figure 12

Trehalose treatment enhances functional recovery in motor and cognitive tasks and decreases depressive-like behavior. (a) Diagram illustrating timepoints by weeks pre- or post-injury and of repeated or terminal behavior experiments conducted on aged mice with Trehalose or Sucrose (i.e., vehicle control) treatment throughout study 2. (b) Graph depicting body weight data of aged mice that underwent continuous Trehalose treatment or vehicle control at baseline and follow-up measurements up to $8 \mathrm{w}$ post-injury. (c) Rotarod data shows decreased latency time to fall in aged mice consuming Sucrose after injury, however no injury effect could be observed in the Trehalose group. (d-g) Spontaneous activity in the open field experiment showed no significant injury effects in the Sucrose group for total distance travelled (e) and average speed (f), however, no neurological deficits were observed for the Trehalose group. (h-i) Y-maze experiment showed significant decrease of arm entry number at $1 \mathrm{w}$ post-injury, but no treatment effect in the percentage of \% alternations. ( $j-k$ ) NOR task showed no group differences in exploration time of left versus right objects during the sample phase, but significant injury effects could 
be observed in the TBI Sucrose group during the choice phase of NOR experiment. (i) The latency to reach food in the center of a novel arena was recorded for the NSF test. The Trehalose TBI group showed significantly lower Open Field versus Home cage ratio when compared to the Sucrose TBI group. No difference was found in latency time in the home cage, confirming that the novel environment was a source of anxiety for TBI mice. (m-o) Graphs depicting the preference index of each sequence for the social recognition experiment. In sequence 3, The TBI Sucrose group failed to show preference for the second, novel stranger mouse, which suggests neurological deficits in social interaction (o). $\mathrm{N}=8$ (Sham Sucrose), 8 (Sham Trehalose), 11 (TBI Sucrose), and 12 (TBI Trehalose). 2-way ANOVA followed by

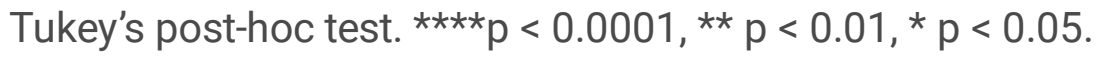
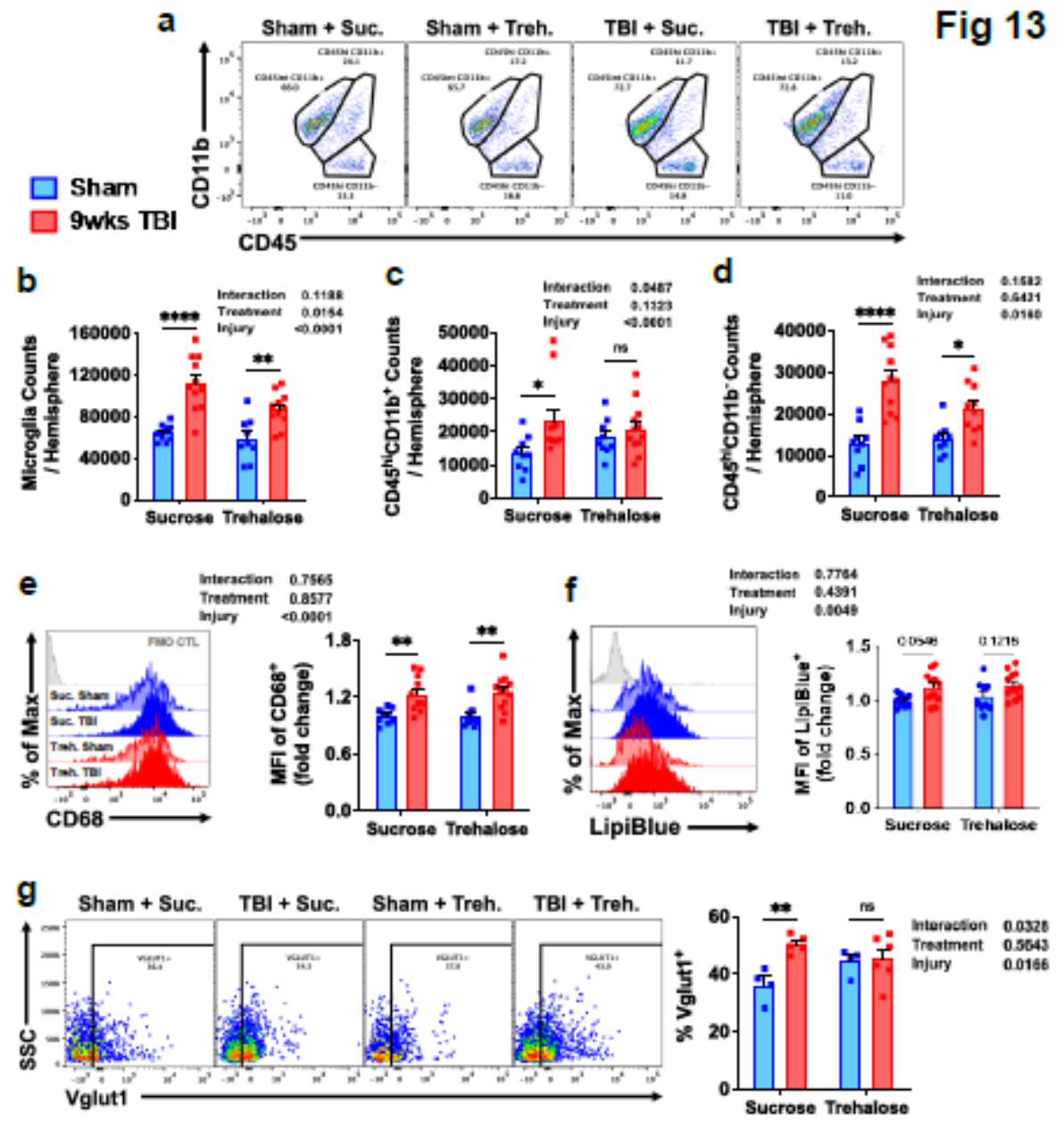

h

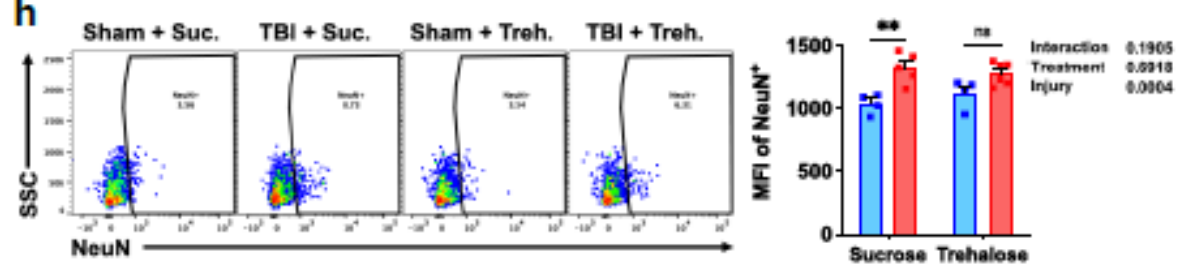

Figure 13

Trehalose treatment reduces long-term microgliosis, lymphocyte infiltration, and phagocytosis of neurons following TBI. (a) A representative dot plot of leukocyte populations in the brain at $9 \mathrm{w}$ after TBI.

Quantification of CD45intCD11b+ microglia (b), CD45hiCD11b+ myeloid cells (c), and CD45hiCD11b- 
putative lymphocyte (d) cell counts are shown. Representative histograms show the relative level of (e) CD68 protein expression and (f) LipiBlue-stained lipid bodies in microglia. (g) The percentage of Vglut1positive microglia in each treatment group is quantified. (h) Representative dot plots illustrate the percentage of NeuN-positive microglia in the ipsilateral hemisphere at $8 \mathrm{w}$ post-TBI. The mean fluorescence intensity of NeuN immunoreactivity is quantified. For all histograms, gray = FMO control, Sucrose $($ vehicle) treated $=$ Blue, Trehalose treated $=$ Red, Sham controls $=$ no fill, and TBI groups $=$ bold fill. N=9-10/group (a-f) and N=4-6/group (g-h). Data were analyzed using 2-way ANOVA group analysis with Tukey's test for multiple comparisons. *** $p<0.0001$, ** $p<0.01$, * $p<0.05$.

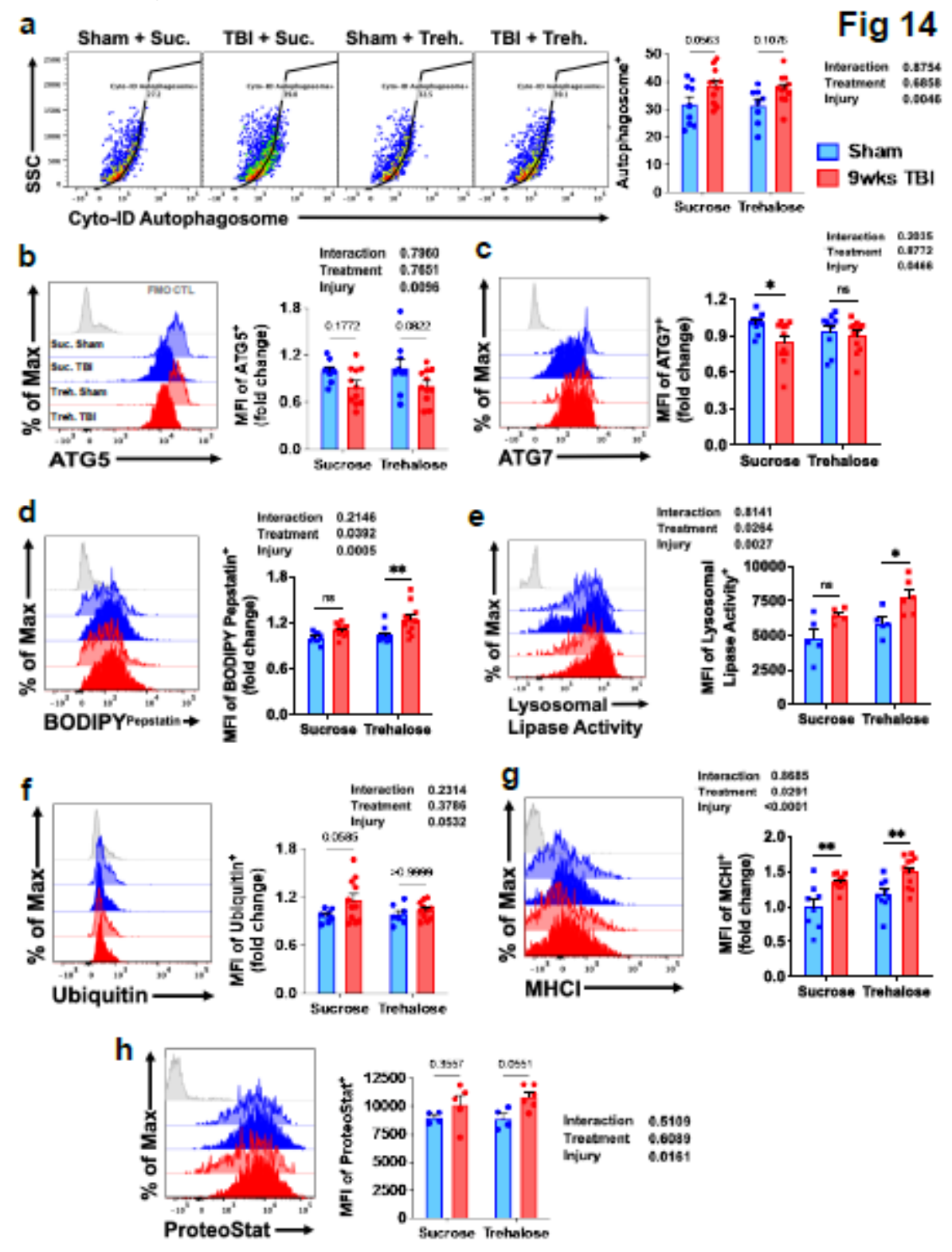

Figure 14

Trehalose treatment modulates multiple steps of the autophagy pathway in microglia. (a) A representative dot plot depicts the percentage of LC3II-positive autophagosomes in microglia at 9w after TBI. Representative histograms show the relative protein expression level of the autophagy-related proteins (b) ATG5 and (c) ATG7 in microglia. Significant effects of Trehalose treatment were seen in 
fluorescence intensity of (d) BODIPYPepstatin and (e) lysosomal lipase activity. Mean fluorescence intensities for ( $f$ ) ubiquitin and $(\mathrm{g}) \mathrm{MHCl}$ are quantified. (h) A group effect of injury was demonstrated in cytosolic protein aggregation as was measured using Proteostat dye. For all histograms, gray = FMO control, Sucrose (vehicle) treated $=$ Blue, Trehalose treated $=$ Red, Sham controls $=$ no fill, and TBI groups = bold fill. $\mathrm{N}=9-10 /$ group (a-d and $\mathrm{f}-\mathrm{g}$ ) and $\mathrm{N}=4-6 /$ group (e and $\mathrm{h}$ ). Data were analyzed using 2-way ANOVA group analysis with Tukey's test for multiple comparisons. ${ }^{\star *} p<0.01,{ }^{*} p<0.05$.

\section{Supplementary Files}

This is a list of supplementary files associated with this preprint. Click to download.

- FigS1.pdf

- Figs2.pdf

- FigS3.pdf

- FigS4.pdf

- Figs5.pdf

- FigS6.pdf 